\title{
The First ANDES Elements: 9-DOF Plate Bending Triangles
}

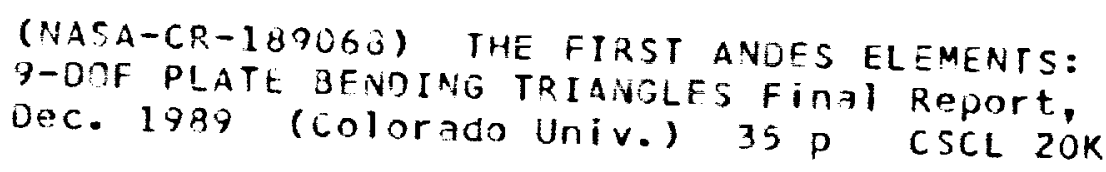

Carmelo Militello and Carlos A. Felippa

University of Colorado

Boulder, Colorado

November 1991

November 1991

Prepared for

Lewis Research Center

Under Grant NAG3-934 



\title{
THE FIRST ANDES ELEMENTS: 9-DOF PLATE BENDING TRIANGLES
}

\author{
CARMELO MILITELLO \\ CARLOS A. FELIPPA \\ Department of Aerospace Engineering Sciences \\ and Center for Space Structures and Controls \\ University of Colorado \\ Boulder, Colorado 80909-0429, USA
}

\begin{abstract}
SUMMARY
New elements are derived to validate and assess the assumed natural deviatoric strain (ANDES) formulation. This is a brand new variant of the assumed natural strain (ANS) formulation of finite elements, which has recently attracted attention as an effective method for constructing high-performance elements for linear and nonlinear analysis. The ANDES formulation is based on an extended parametrized variational principle developed in recent publications. The key concept is that only the deviatoric part of the strains is assumed over the element whereas the mean strain part is discarded in favor of a constant stress assumption. Unlike conventional ANS elements, ANDES elements satisfy the individual element test (a stringent form of the patch test) a priori while retaining the favorable distortion-insensitivity properties of ANS elements. The first application of this new formulation is the development of several Kirchhoff plate bending triangular elements with the standard nine degrees of freedom. Linear curvature variations are sampled along the three sides with the corners as "gage reading" points. These sample values are interpolated over the triangle using three schemes. Two schemes merge back to conventional ANS elements, one being identical to the Discrete Kirchhoff Triangle (DKT), whereas the third one produces two new ANDES elements. Numerical experiments indicate that one of the ANDES element is relatively insensitive to distortion compared to previously derived high-performance plate-bending elements, while retaining accuracy for nondistorted elements.
\end{abstract}




\section{INTRODUCTION}

Despite almost three decades of work, plates and shells remain a important area of research in finite element methods. Challenging topics include:

1. The construction of high performance elements.

2. The modeling of composite and stiffened wall constructions.

3. The treatment of prestress, imperfections, nonlinear, dissipative and dynamic effects.

4. The development of practical error estimators and adaptive discretization methods.

5. The interaction with nonstructural components, for example external and internal fluids.

This paper addresses primarily the first challenge, although it must be recognized that progress in this direction is shaped to some extent by thinking of the others. The main motivation here is the construction of simple and efficient finite elements for plates and shells that are lock-free, rank sufficient and distortion insensitive, yield accurate answers for coarse meshes; fit naturally into displacement-based programs, and can be easily extended to nonlinear and dynamic problems. Elements that possess these attributes to some noticeable degree are collectively known as high performance or HP elements.

Over the past three decades investigators have resorted to many ingenious devices to construct HP elements. The most important ones are listed in Table 1. The underlying theme is that although the final product may look like a standard displacement model so as to fit naturally into existing finite element programs, the conventional displacement formulation is abandoned. (By "conventional" we mean the use of conforming displacement assumptions into the total potential energy principle.)

\subsection{A Unified Variational Framework}

Table 1 conveys the feeling of a bewildering array of tools. The question arises as to whether some of them are just facets of the same thing. Limited progress has been made in this regard. One notable advance in the 1970s has been the equivalence of reduced/selective integration and mixed methods achieved by Malkus and Hughes [25].

The present work has benefited from the unplanned confluence of two unification efforts. An initial attempt to place the free formulation [6-9] within the framework of parametrized hybrid variational principles was successful [14-16]. The free formulation in turn "dragged" incompatible shape functions, the patch test. and energy balancing into the scene. Concurrently a separate effort was carried out to set out the assumed natural strain (ANS) and projection methods in a mixed/hybrid variational framework [26,27]. Comparison of the results led to the rather unexpected conclusion that a parametrized variational framework was able to encompass ANS and the free formulation as well as some hitherto untried methods $[17,18]$.

The common theme emerging from this unification is that a wide class of HP elements can be constructed using two ingredients:

(1) A parametrized functional that contains all variational principles of elasticity as special cases.

(2) Additional assumptions (sometimes called "variational crimes" or "tricks") that can be placed on a variational setting through Lagrange multipliers.

As of this writing it is not known whether the "wide class" referred to above encompasses all HP elements or at least the most interesting ones. Some surprising coalescences, such as DKT and ANS bending elements, however, have emerged from this study. 
Table 1. Tools for Constructing HP Elements

\begin{tabular}{|llr|}
\hline & Technique & Year introduced \\
1. & Incompatible shape functions & early $1960 \mathrm{~s}$ \\
2. & Patch test & 1965 \\
3. & Mixed and hybrid variational principles & 1965 \\
4. & Projectors & 1967 \\
5. & Selective reduced integration & 1969 \\
6. & Uniform reduced integration & 1970 \\
7. & Partial strain assumptions & 1970 \\
8. & Energy balancing & 1974 \\
9. & Directional integration & 1978 \\
10. & Limit differential equations. & 1982 \\
11. & Free formulation & 1984 \\
12. & Assumed natural strains & 1984 \\
\hline
\end{tabular}

\subsection{The Assumed Natural Strain Formulation}

The assumed natural strain (ANS) formulation of finite elements is a relatively new development. A restricted form of the assumed strain method (not involving natural strains) was introduced in 1969 by Willam [33], who constructed a 4-node plane-stress element by assuming a constant shear strain independently of the direct strains and using a strain-displacement mixed variational principle. (The resulting element is identical to that derivable by selective one-point integration.) A different approach advocated by Ashwell [1] and coworkers viewed "strain elements" as a way to obtain appropriate displacement fields by integration of appropriately assumed compatible strain fields.*

These and other forms of assumed-strain techniques were overshadowed in the 1970 s by developments in reduced and selective integration methods. The assumed strain approach in natural coordinates, however, has recently attracted substantial attention $[2,11,19,22,24,29,30,31]$, particularly in view of its effectiveness in geometrically nonlinear analysis.

As noted above, the unification presented in $[17,18]$ merges two HP element construction schemes: the free formulation (FF) of Bergan and Nygård [8] and a variant of ANS called ANDES (acronym for Assumed Natural Deviatoric Strains) described in further detail below. The stiffness equations produced by the unified formulation enjoy the fundamental decomposition property summarized in Box 1 .

In the ANDES variant of ANS, assumptions are made only on the deviatoric portion of the element strains. namely that portion that integrates to zero over each element. This assumption produces the higher order stiffness labeled $K_{h 22}$ in Box 1 . The mean portion of the strains is left to be determined variationally from assumptions on the limit stress field, and has no effect on the stiftness equations.

* In fact, this was the technique originally used by Turner et al. [32] for deriving the constant-strain membrane triangle in their celebrated 1956 paper. 
This paper describes the construction of the first ANDES elements. These are Kirchhoff plate-bending triangular elements with the standard 9 degrees of freedom (one displacement and two rotations at each corner). This choice is made because of the following reasons:

1. High-performance three-node triangular plate bending elements, whether based on Kirchhoff or ReissnerMindlin mathematical models, have not been previously obtained through the ANS formulation. (Although the DKT element [3] qualifies as high-performance and is in fact an ANS element as shown later, it has not been derived as such.) The situation is in sharp contrast to four-node quadrilateral bending elements, for which HP elements have been constructed through a greater variety of tools; see e.g. $[11,20,23,24,29]$.

2. High performance elements of this type have been obtained through the FF and ancestors of the FF $[6,7,8,12]$, and they are considered among the best performers available. It is therefore intriguing whether elements based on the ANDES variant can match or exceed this performance.

The basic steps in the construction of $\mathbf{K}_{b}$ and $\mathbf{K}_{h}$ for a general three-dimensional element are summarized in Boxes 2 and 3, respectively. For justification of these "recipees" the reader is referred to $[17,18]$. The derivation of conventional ANS elements is summarized in Box 4. 


\section{Box 1 Decomposition of the Element Stiffness Equations}

Let $\mathbf{K}$ be the element stiffness matrix, $\mathbf{v}$ the visible element degrees of freedom (those degrees of freedom in common with other elements, also called the connectors) and $f$ the corresponding element node forces. Then the element stiffness equations decompose as

$$
\mathbf{K} \mathbf{v}=\left(\mathbf{K}_{b}+\mathbf{K}_{h}\right) \mathbf{v}=\mathbf{f} .
$$

$\mathbf{K}_{b}$ and $\mathbf{K}_{h}$ are called the basic and higher order stiffness matrices, respectively. The basic stiffness matrix, which is usually rank deficient, is constructed for convergence. The higher order stiffness matrix is constructed for stability and (in more recent work) accuracy. A decomposition of this nature, which also holds at the assembly level, was first obtained by Bergan and Nygard in the derivation of the free formulation [8].

In the unified formulation presented in $[17,18]$ the following key properties of the decomposition (1) are derived.

1. $\mathbf{K}_{\mathbf{b}}$ is formulation independent and is defined entirely by an assumed constant stress state working on element boundary displacements. No knowledge of the interior displacements is necessary (Box 2). The extension of this statement to $C^{0}$ plate and shell elements is not straightforward, however, and special considerations are necessary in order to obtain $\mathbf{K}_{\mathbf{b}}$ for those elements.

2. $\mathbf{K}_{h}$ has the general form

$$
\mathbf{K}_{h}=j_{33} K_{h 33}+j_{22} K_{h 22}+j_{23} K_{h 23} .
$$

The three parameters $j_{22}, j_{23}$ and $j_{33}$ characterize the source variational principle in the following sense:

(a) The FF is recovered if $j_{22}=j_{23}=0$ and $j_{33}=1-\gamma$, where $\gamma$ is a $K_{h}$ scaling coefficient studied in $[9,13]$. The original FF of $[8]$ is obtained if $\gamma=0$. The source variational principle is a one-parameter form that includes the potential energy and stress-displacement Reissner functionals as special cases [14-16].

(b) The ANDES variant of ANS is recovered if $j_{22}=j_{23}=0$ whereas $j_{22}=\alpha$ is a scaling parameter. The source variational principle is a one-parameter form that includes Reissner's stress-displacement and Hu-Washizu's functionals as special cases [18].

(c) If $j_{23}$ is nonzero, the last term in (2) may be viewed as being produced by a FF/ANDES combination. Such a combination remains unexplored. 


\section{Box 2 Construction of the Basic Stiffness Matrix $\mathbf{K}_{b}$}

Step B.1. Assume a constant stress field, $\bar{\sigma}$, inside the element. (This should be the element stress field that holds in the convergence limit; for structural elements the assumption would be on independent stress resultants. ) The associated boundary tractions are $\bar{\sigma}_{n}=$ $\bar{\sigma} . \mathbf{n}$, where $\mathbf{n}$ denotes the unit external normal on the element boundary $S$.

Step B.2. Assume boundary displacements, d, over $S$. This field is described in terms of the visible element node displacements $v$ (also called the connectors) as

$$
\mathbf{d}=\mathbf{N}_{d} \mathbf{v},
$$

where $\mathrm{N}_{d}$ is an array of boundary shape functions. The boundary motions (3) must satisfy interelement continuity (or at least, zero mean discontinuity so that no energy is lost at interfaces) and contain rigid-body and constant-strain motions exactly.

Step B.9. Construct the "lumping matrix" L that consistently "lumps" the boundary tractions $\bar{\sigma}_{n}$ into element node forces, $\bar{f}$, conjugate to $v$ in the virtual work sense. That is,

$$
\overline{\mathbf{f}}=\int_{S} \mathrm{~N}_{d n} \bar{\sigma}_{n} d S=\mathbf{L} \bar{\sigma}
$$

In the above, $\mathbf{N}_{d n}$ are boundary-system projections of $\mathbf{N}_{d}$ conjugate to the surface tractions $\bar{\sigma}_{n}$.

Step B.4. The basic stiffness matrix for a 3D element

$$
\mathrm{K}_{b}=v^{-1} \mathrm{LEL}^{T},
$$

where $\mathbf{E}$ is the stress-strain constitutive matrix of elastic moduli, which are assumed to be constant over the element, and $v=\int_{V} d V$ is the element volume measure.

For a Kirchhoff plate bending element, stresses, strains and stress-strain moduli become bending moments, curvatures and moment-curvature moduli, respectively, and the integration is performed over the element area $A$ :

$$
\mathbf{K}_{b}=A^{-1} \mathbf{L D L}^{T}
$$

where $\mathbf{D}$ is the matrix of moment-curvature moduli. Specific examples for $\mathbf{L}$ are provided in Section 4. 


\section{Box 3 Construction of $K_{h}$ by the ANDES Formulation}

Step H.1. Select reference lines (in 2D elements) or reference planes (in 3D elements) where "natural straingage" locations are to be chosen. By appropriate interpolation express the element natural strains $\epsilon$ in terms of the "straingage readings" $g$ at those locations:

$$
\epsilon=A_{\epsilon} \mathbf{g}
$$

where $\epsilon$ is a strain field in natural coordinates that must include all constant strain states. (For structural elements the term "strain" is to be interpreted in a generalized sense.)

Step H.2. Relate the Cartesian strains e to the natural strains:

$$
\mathrm{e}=\mathrm{T} \epsilon=\mathrm{TA}_{\mathrm{c} g} \mathrm{~g}=\mathrm{Ag}
$$

at each point in the element. (If $\mathbf{e} \equiv \epsilon$, or if it is possible to work throughout in natural coordinates, this step is skipped.)

Step H.S. Relate the natural straingage readings $\mathbf{g}$ to the visible degrees of freedom

$$
\mathbf{g}=\mathrm{Q} \mathbf{v}
$$

where $Q$ is a straingage-to-node displacement transformation matrix. Techniques for doing this vary from element to element and it is difficult to state rules that apply to every situation. In the elements derived here $Q$ is constructed by direct interpolation over the reference lines. (In general there is no unique internal displacement field $\mathbf{u}$ whose symmetric gradient is $\mathbf{e}$ or $\epsilon$, so this step cannot be done by simply integrating the strain field over the element and collocating $\mathbf{u}$ at the nodes.)

Step H.4. Split the Cartesian strain field into mean (volume-averaged) and deviatoric strains:

$$
\mathbf{e}=\overline{\mathbf{e}}+\mathbf{e}_{d}=\left(\overline{\mathbf{A}}+\mathbf{A}_{d}\right) \mathbf{g},
$$

where $\overline{\mathbf{A}}=\int_{V} \mathbf{T} \mathbf{A}_{\epsilon} d V / v$, and $\mathbf{e}_{d}=\mathbf{A}_{d} \mathbf{g}$ has mean zero value over $V$. This step may also be carried out on the natural strains if $\mathbf{T}$ is constant, as is the case for the elements here.

Step H.5. The higher-order stiffness matrix is given by

$$
\mathbf{K}_{h}=\alpha \mathbf{Q}^{T} \mathbf{K}_{d} \mathbf{Q}, \quad \text { with } \quad \mathbf{K}_{d}=\int_{V} \mathbf{A}_{d}^{T} \mathbf{E} \mathbf{A}_{d} d V,
$$

where $\alpha=j_{22}>0$ is a scaling coefficient (see Box 1).

It is often convenient to combine the product of $A$ and $Q$ into a single strain-displacement matrix called (as usual) $\mathbf{B}$, which splits into $\overline{\mathbf{B}}$ and $\mathbf{B}_{d}$ :

$$
\mathbf{e}=\mathbf{A Q v}=\left(\overline{\mathbf{A}}+\mathbf{A}_{d}\right) \mathbf{Q} \mathbf{v}=\left(\overline{\mathbf{B}}+\mathbf{B}_{d}\right) \mathbf{v}=\mathbf{B} \mathbf{v},
$$

in which case

$$
\mathbf{K}_{h}=\int_{V} \mathbf{B}_{d}^{T} \mathbf{E B}_{d} d V .
$$

The notation $\mathbf{B}_{\epsilon}=\mathbf{A}_{\epsilon} \mathbf{Q}$ is also used in the sequel. 


\section{Box 4 Construction of $\mathrm{K}$ by the Conventional ANS Formulation}

Steps S.I to S.9. Identical to the first three steps H.I through H.9, in Box 3. The fourth step: strain splitting, is omitted.

Step S.4. The element stiffness matrix is given by

$$
\mathbf{K}=\mathbf{Q}^{T} \mathbf{K}_{a} \mathbf{Q}, \quad \text { with } \quad \mathbf{K}_{a}=\int_{V} \mathbf{A}^{T} \mathbf{E A} d V
$$

or, if $\mathbf{B}=\mathbf{A} \mathbf{Q}$ is readily available

$$
\mathbf{K}=\int_{V} \mathbf{B}^{T} \mathbf{E B} d V
$$

In general this stiffness matrix does not pass the individual element test of Bergan and Hanssen [6,7] (a strong form of the patch test that demands pairwise cancellation of node forces between adjacent elements in constant stress states). For this to happen, $\mathbf{K}$ must admit the decomposition

$$
\mathbf{K}=\mathbf{K}_{b}+\mathbf{K}_{h}=v^{-1} \mathbf{L E L}^{T}+\mathbf{K}_{h},
$$

where $\mathbf{L}$ is a force-lumping matrix derivable as per Box 2 and $\mathbf{K}_{h}$ is orthogonal to the rigid body and constant strain test motions. In other words, the ANS element must coalesce with the ANDES formulation with $\alpha=1$. The equivalence may be checked by requiring that

$$
\overline{\mathbf{B}}=\overline{\mathbf{A}} \mathbf{Q}=v^{-1} \mathbf{L}^{T},
$$

where $\overline{\mathbf{A}}$ is the mean part of $\mathbf{A}$. At the present there are no general techniques for explicit construction of strain fields that satisfy these conditions a priori.

If the patch test is not satisfied, one should switch to the ANDES formulation by replacing the basic stiffness constructed from constant strain, namely $v \overline{\mathbf{B}}^{T} \mathbf{E} \overline{\mathbf{B}}$, with one constructed from constant stress as in Box 2.

\section{THE TRIANGULAR ELEMENT}

\subsection{Geometric Relations}

The geometry of an individual triangle is illustrated in Figure 1. The triangle has straight sides. Its geometry is completely defined by the location of its three corners, which are labelled $1,2,3$, traversed counterclockwise. The element is referred to a local Cartesian system $(x, y)$ which is usually taken with origin at the centroid 0 , whence the corner coordinates $x_{i}, y_{i}$ satisfy the relations

$$
x_{1}+x_{2}+x_{3}=0, \quad y_{1}+y_{2}+y_{3}=0 .
$$

Coordinate differences are abbreviated by writing $x_{i j}=x_{i}-x_{j}$, and $y_{i j}=y_{i}-y_{j}$. The signed triangle area $A$ is given by

$$
2 A=\left|\begin{array}{ccc}
1 & 1 & 1 \\
x_{1} & x_{2} & x_{3} \\
y_{1} & y_{2} & y_{3}
\end{array}\right|=x_{21} y_{31}-x_{31} y_{21}=x_{32} y_{12}-x_{12} y_{32}=x_{13} y_{23}-x_{23} y_{13}
$$




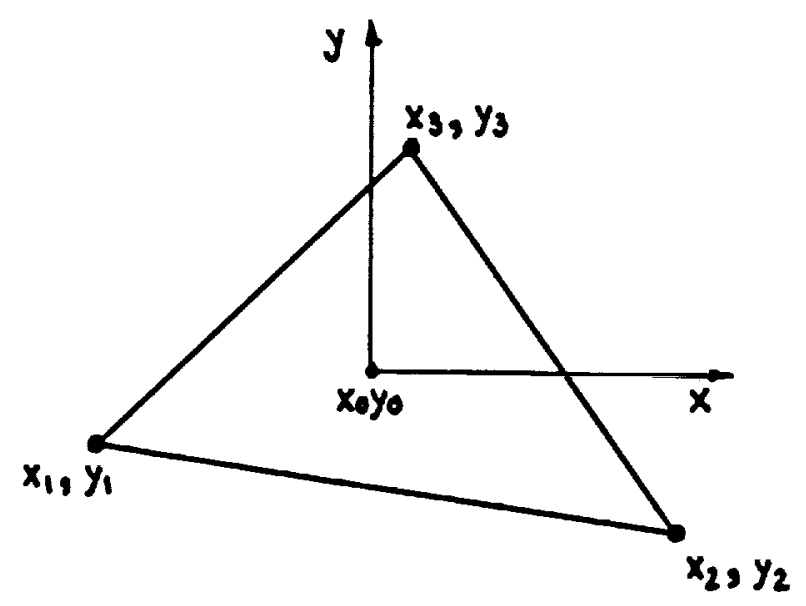

Figure 1 The triangular element

and we require that $A>0$. We shall make use of dimensionless triangular coordinates $\zeta_{1}, \zeta_{2}$ and $\zeta_{3}$, linked by $\zeta_{1}+\zeta_{2}+\zeta_{3}=1$. The following well known relations between the triangular and Cartesian coordinates of a straight-sided triangle are noted for further use:

$$
\begin{aligned}
& x=x_{1} \zeta_{1}+x_{2} \zeta_{2}+x_{3} \zeta_{3}, \quad y=y_{1} \zeta_{1}+y_{2} \zeta_{2}+y_{3} \zeta_{3}, \\
& \zeta_{i}=\frac{1}{2 A}\left[x_{i} y_{k}-x_{k} y_{j}+\left(x-x_{0}\right) y_{j k}+\left(y-y_{0}\right) x_{k j}\right]
\end{aligned}
$$

in which $i, j$ and $k$ denote positive cyclic permutations of 1,2 and 3 ; for example, $i=2, j=3, k=1$. (If the origin is taken at the centroid as in Figure 1, $x_{0}=y_{0}=0$.) It follows that

$$
\begin{array}{lll}
2 A \frac{\partial \zeta_{1}}{\partial x}=y_{23}, & 2 A \frac{\partial \zeta_{2}}{\partial x}=y_{31}, & 2 A \frac{\partial \zeta_{3}}{\partial x}=y_{12} \\
2 A \frac{\partial \zeta_{1}}{\partial y}=x_{32}, & 2 A \frac{\partial \zeta_{2}}{\partial y}=x_{13}, & 2 A \frac{\partial \zeta_{3}}{\partial y}=x_{21} .
\end{array}
$$

Other intrinsic dimensions and ratios of use in future derivations are (see Figure 2)

$$
\begin{aligned}
\ell_{i j} & =\ell_{j i}=\sqrt{x_{i j}^{2}+y_{i j}^{2}}, \quad c_{i j}=x_{j i} / \ell_{i j}, \quad s_{i j}=y_{j i} / \ell_{i j}, \\
a_{k} & =2 A / \ell_{i j}, \quad b_{i j}=\left(x_{i j} x_{i k}+y_{j i} y_{k i}\right) / \ell_{i j}=\ell_{i j}-b_{j i}, \\
\lambda_{i j} & =b_{i j} / \ell_{i j}=\left(x_{i j} x_{i k}+y_{j i} y_{k i}\right) /\left(x_{i j}^{2}+y_{i j}^{2}\right), \quad \lambda_{j i}=1-\lambda_{i j}=b_{j i} / \ell_{i j} .
\end{aligned}
$$

Here $\ell_{i j}=\ell_{j i}$ is the length of side $i-j$ and $c_{i j}$ and $s_{i j}$ the cosine and sine, respectively, of angle $(i-j, x)$. Furthermore $b_{i j}$ and $b_{j i}$ are the projections of sides $i-k$ and $k-j$, respectively, onto $i-j ; \lambda_{i j}$ and $\lambda_{j i}$ being the corresponding projection ratios.

On each side $i-j$, define the dimensionless natural coordinates $\mu_{i j}$ as varying from 0 at $i$ to 1 at $j$. The coordinate $\mu_{i j}$ of a point not on the side is that of its projection on $i-j$. Obviously

$$
\frac{\partial x}{\partial \mu_{i j}}=x_{j i}, \quad \frac{\partial y}{\partial \mu_{i j}}=y_{j i}
$$






Figure 2 Intrinsic dimensions of triangle

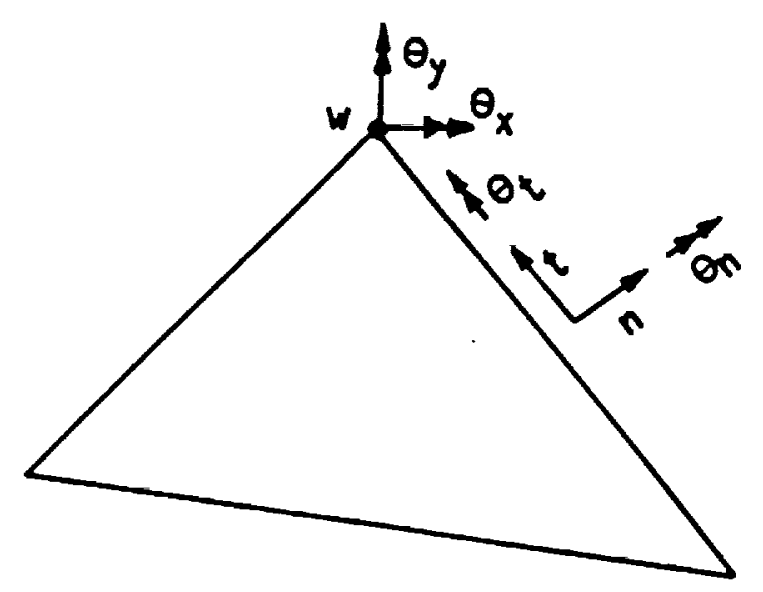

Figure 3 Local coordinate systems over a element side 


\subsection{Displacements, Rotations, Curvatures}

As we are dealing with a Kirchhoff element, its displacement field is completely defined by the transverse displacement $w(x, y) \equiv w\left(\zeta_{1}, \zeta_{2}, \zeta_{3}\right)$, positive upwards. In the present section we assume that $w$ is unique and known inside the element; this assumption is relaxed later. The midplane (covariant) rotations about $x$ and $y$ are $\theta_{x}=\partial w / \partial y$ and $\theta_{y}=-\partial w / \partial x$, respectively. Along side $i-j$ with tangential direction $t$ and external-normal $\mathbf{n}$ (see Figure 3 ) the tangential and normal rotations are defined as

$$
\begin{aligned}
\theta_{n} & =\frac{\partial w}{\partial t}=\theta_{x} s_{i j}-\theta_{y} c_{i j}, \\
\theta_{i} & =-\frac{\partial w}{\partial n}=\theta_{x} c_{i j}+\theta_{y} s_{i j} .
\end{aligned}
$$

The visible degrees of freedom of the element collected in $\mathbf{v}$ (see Boxes 2-3) are

$$
\mathbf{v}^{T}=\left[\begin{array}{lllllllll}
w_{1} & \theta_{x 1} & \theta_{y 1} & w_{2} & \theta_{x 2} & \theta_{y 2} & w_{3} & \theta_{x 3} & \theta_{y 3}
\end{array}\right]
$$

The first and second derivatives of the displacement $w$ with respect to the Cartesian and triangular coordinates are linked by the relations (summation convention used)

$$
\begin{gathered}
\frac{\partial w}{\partial x}=\frac{\partial w}{\partial \zeta_{i}} \frac{\partial \zeta_{i}}{\partial x}=\frac{1}{2 A} \frac{\partial w}{\partial \zeta_{i}} y_{j k}, \\
\frac{\partial w}{\partial y}=\frac{\partial w}{\partial \zeta_{i}} \frac{\partial \zeta_{i}}{\partial y}=\frac{1}{2 A} \frac{\partial w}{\partial \zeta_{i}} x_{k j} \\
\frac{\partial^{2} w}{\partial x^{2}}=\frac{\partial^{2} w}{\partial \zeta_{i} \partial \zeta_{j}} \frac{\partial \zeta_{i}}{\partial x} \frac{\partial \zeta_{j}}{\partial x}+\frac{\partial w}{\partial \zeta_{i}} \frac{\partial^{2} \zeta_{i}}{\partial x^{2}}=\frac{1}{4 A^{2}} \frac{\partial^{2} w}{\partial \zeta_{i} \partial \zeta_{j}} y_{j k} y_{k i}, \\
\frac{\partial^{2} w}{\partial x \partial y}=\frac{\partial^{2} w}{\partial \zeta_{i} \partial \zeta_{j}} \frac{\partial \zeta_{i}}{\partial x} \frac{\partial \zeta_{j}}{\partial y}+\frac{\partial w}{\partial \zeta_{i}} \frac{\partial^{2} \zeta_{i}}{\partial x \partial y}=\frac{1}{4 A^{2}} \frac{\partial^{2} w}{\partial \zeta_{i} \partial \zeta_{j}} y_{j k} x_{i k} \\
\frac{\partial^{2} w}{\partial y^{2}}=\frac{\partial^{2} w}{\partial \zeta_{i} \partial \zeta_{j}} \frac{\partial \zeta_{i}}{\partial y} \frac{\partial \zeta_{j}}{\partial y}+\frac{\partial w}{\partial \zeta_{i}} \frac{\partial^{2} \zeta_{i}}{\partial y^{2}}=\frac{1}{4 A^{2}} \frac{\partial^{2} w}{\partial \zeta_{i} \partial \zeta_{j}} x_{k j} x_{i k}
\end{gathered}
$$

since $\partial^{2} \zeta_{i}^{2} / \partial x^{2}, \partial^{2} \zeta_{i}^{2} / \partial x \partial y$ and $\partial^{2} \zeta_{i}^{2} / \partial y^{2}$ vanish on a straight-sided triangle, cf. Eq. (22). We can represent the second derivative relations in matrix form as

$$
\left\{\begin{array}{c}
\frac{\partial^{2} w}{\partial x^{2}} \\
\frac{\partial^{2} w}{\partial y^{2}} \\
2 \frac{\partial^{2} w}{\partial x \partial y}
\end{array}\right\}=\frac{1}{4 A^{2}}\left[\begin{array}{ccc}
y_{23}^{2} & x_{32}^{2} & 2 x_{32} y_{23} \\
y_{31}^{2} & x_{13}^{2} & 2 x_{13} y_{31} \\
y_{12}^{2} & x_{21}^{2} & 2 x_{21} y_{12} \\
2 y_{23} y_{31} & 2 x_{32} x_{13} & x_{32} y_{31}+x_{13} y_{23} \\
2 y_{31} y_{12} & 2 x_{13} x_{21} & x_{13} y_{12}+x_{21} y_{31} \\
2 y_{12} y_{23} & 2 x_{21} x_{32} & x_{21} y_{23}+x_{32} y_{12}
\end{array}\right]^{T}\left[\begin{array}{c}
\frac{\partial^{2} w}{\partial \zeta_{1}^{2}} \\
\frac{\partial^{2} w}{\partial \zeta_{2}^{2}} \\
\frac{\partial^{2} w}{\partial \zeta_{3}^{2}} \\
\frac{\partial^{2} w}{\partial \zeta_{1} \partial \zeta_{2}} \\
\frac{\partial^{2} w}{\partial \zeta_{2} \partial \zeta_{3}} \\
\frac{\partial^{2} w}{\partial \zeta_{3} \partial \zeta_{1}}
\end{array}\right\}
$$

or

$$
\kappa=\mathbf{w}_{\zeta \zeta}
$$

The inverse relation does not exist. 


\subsection{Natural Curvatures}

The second derivatives of $w$ with respect to the dimensionless side directions defined in Section 2.1 will be called the natural curvatures and denoted by $\chi_{i j}=\partial^{2} w / \partial \mu_{i j}^{2}$. Note that these curvatures have dimensions of displacement. The natural curvatures can be related to the Cartesian plate curvatures $\kappa_{x x}=\partial^{2} w / \partial x^{2}$, $\kappa_{y y}=\partial^{2} w / \partial y^{2}$ and $\kappa_{x y}=2 \partial^{2} w / \partial x \partial y$, by chain-rule application of (22):

$$
\chi=\left\{\begin{array}{l}
\chi_{12} \\
\chi_{23} \\
\chi_{31}
\end{array}\right\}=\left\{\begin{array}{c}
\frac{\partial^{2} w}{\partial \mu_{12}^{2}} \\
\frac{\partial^{2} w}{\partial \mu_{23}^{2}} \\
\frac{\partial^{2} w}{\partial \mu_{31}^{2}}
\end{array}\right\}=\left[\begin{array}{lll}
x_{21}^{2} & y_{21}^{2} & x_{21} y_{21} \\
x_{32}^{2} & y_{32}^{2} & x_{32} y_{32} \\
x_{13}^{2} & y_{13}^{2} & x_{13} y_{13}
\end{array}\right]\left\{\begin{array}{c}
\frac{\partial^{2} w}{\partial x^{2}} \\
\frac{\partial^{2} w}{\partial y^{2}} \\
2 \frac{\partial^{2} w}{\partial x \partial y}
\end{array}\right\}=\mathbf{T}^{-1} \kappa
$$

The inverse of this relation is

$$
\left\{\begin{array}{c}
\frac{\partial^{2} w}{\partial x^{2}} \\
\frac{\partial^{2} w}{\partial y^{2}} \\
2 \frac{\partial^{2} w}{\partial x \partial y}
\end{array}\right\}=\frac{1}{4 A^{2}}\left[\begin{array}{ccc}
y_{23} y_{13} & y_{31} y_{21} & y_{12} y_{32} \\
x_{23} x_{13} & x_{31} x_{21} & x_{12} x_{32} \\
y_{23} x_{31}+x_{32} y_{13} & y_{31} x_{12}+x_{13} y_{21} & y_{12} x_{23}+x_{21} y_{32}
\end{array}\right]\left\{\begin{array}{l}
\frac{\partial^{2} w}{\partial \mu_{12}^{2}} \\
\frac{\partial^{2} w}{\partial \mu_{23}^{2}} \\
\frac{\partial^{2} w}{\partial \mu_{31}^{2}}
\end{array}\right\},
$$

or, in compact matrix notation

$$
\boldsymbol{\kappa}=\mathbf{T} \boldsymbol{\chi} .
$$

A comparison of (29) with (31)-(32) displays the advantages of natural curvatures over triangle-coordinate curvatures when the curvature field is to be constructed directly. On the other hand, (29) is useful when the transverse displacement $w$ over the element is built as a function of the triangular coordinates.

At this point we relax the requirement that the curvatures be derivable from a displacement field $w$; consequently the partial derivative notation will be discontinued. However, the foregoing transformations will be assumed to hold even if the curvature fields $\kappa$ and $\chi$ are not derivable from $w$.

\section{DIRECT CURVATURE INTERPOLATION}

\subsection{The Straingage Readings}

ANS and ANDES plate bending elements are based on direct interpolation of natural curvatures. All elements discussed here adopt the three triangle sides as the reference lines defined in Box 3 . The natural curvatures are assumed to vary linearly over each reference line, an assumption which is obviously consistent with cubic beam-like variations of $w$ over the sides. A linear variation on each side is determined by two straingage sample points, which we chose to be at the corners.

Over each triangle side chose the isoparametric coordinates $\xi_{i j}$ that vary from -1 at corner $i$ to +1 at corner $j$. These are related to the $\mu_{i j}$ coordinates introduced in Section 2.1 by $\xi_{i j}=2 \mu_{i j}-1$. The Hermite interpolation of $w$ over $i-j$ is

$$
w=\frac{1}{4}\left[\begin{array}{llll}
\left(1-\xi_{i j}\right)^{2}\left(2+\xi_{i j}\right) & \frac{1}{2} \ell_{i j}\left(1-\xi_{i j}\right)^{2}\left(1+\xi_{i j}\right) & \left(1+\xi_{i j}\right)^{2}\left(2-\xi_{i j}\right) & -\frac{1}{2} \ell_{i j}\left(1+\xi_{i j}\right)^{2}\left(1-\xi_{i j}\right)
\end{array}\right]\left\{\begin{array}{c}
w_{i} \\
\theta_{n i} \\
u_{j}^{\prime} \\
\theta_{n j}
\end{array}\right\}
$$

where $\theta_{n}$ denotes the rotation about the external normal $n$ on side $i j$. The natural curvature over side $i j$ is given by

$$
\chi_{i j}=\frac{\partial^{2} w}{\partial \mu_{i j}^{2}}=\left[\begin{array}{llll}
6 \xi_{i j} & 3 \ell_{i j}\left(\xi_{i j}-1\right) & -6 \xi_{i j} & 3 \ell_{i j}\left(\xi_{i j}+1\right)
\end{array}\right]\left\{\begin{array}{c}
w_{i} \\
\theta_{n i} \\
w_{j} \\
\theta_{n j}
\end{array}\right\}
$$


Evaluating these relations at the nodes by setting $\xi_{i j}= \pm 1$ and converting normal rotations to $x-y$ rotations through (25), we build the transformation

$$
\left\{\begin{array}{l}
\left.\chi_{12}\right|_{1} \\
\left.\chi_{12}\right|_{2} \\
\left.\chi_{23}\right|_{2} \\
\left.\chi_{23}\right|_{3} \\
\left.\chi_{31}\right|_{3} \\
\left.\chi_{31}\right|_{1}
\end{array}\right\}=\left[\begin{array}{rrrrrrrrr}
-6 & -4 y_{21} & 4 x_{21} & 6 & -2 y_{21} & 2 x_{21} & 0 & 0 & 0 \\
6 & 2 y_{21} & -2 x_{21} & -6 & 4 y_{21} & -4 x_{21} & 0 & 0 & 0 \\
0 & 0 & 0 & -6 & -4 y_{32} & 4 x_{32} & 6 & -2 y_{32} & 2 x_{32} \\
0 & 0 & 0 & 6 & 2 y_{32} & -2 x_{32} & -6 & 4 y_{32} & -4 x_{32} \\
6 & -2 y_{13} & 2 x_{13} & 0 & 0 & 0 & -6 & -4 y_{13} & 4 x_{13} \\
-6 & 4 y_{13} & -4 x_{13} & 0 & 0 & 0 & 6 & 2 y_{13} & -2 x_{13}
\end{array}\right\}\left\{\begin{array}{l}
w_{1} \\
\theta_{x 1} \\
\theta_{y 1} \\
w_{2} \\
\theta_{x 2} \\
\theta_{y 2} \\
w_{3} \\
\theta_{x 3} \\
\theta_{y 3}
\end{array}\right\}
$$

The left hand side is the natural straingage reading vector called $g$ in Box 3 and thus we can express (35) as

$$
\mathbf{g}=\mathbf{Q} \mathbf{v}
$$

This relation holds for all elements discussed here.

The six gage readings collected in g provide curvatures along the three triangle side directions at two corners. But nine values are needed to recover the complete curvature field over the element. The three additional values are the natural curvatures $\chi_{23}, \chi_{31}$ and $\chi_{12}$ at corners 1,2 and 3 , respectively. Three possibilities for the missing values are discussed below.

\subsection{The A verage-Curvature Rule}

To each corner $k$ assign the average natural curvature $\chi_{i j}$ of the opposite side. This average is given by (34) evaluated at $\xi_{i j}=0$. For example

$$
\left.\chi_{12}\right|_{3}=\frac{1}{2}\left(\left.\chi_{12}\right|_{1}+\left.\chi_{12}\right|_{2}\right)=y_{21}\left(\theta_{x 2}-\theta_{x 1}\right)+x_{12}\left(\theta_{y 2}-\theta_{y 1}\right) .
$$

The natural curvature now can be interpolated linearly over the triangle:

$$
\chi_{12}=\left.\chi_{12}\right|_{1} \zeta_{1}+\left.\chi_{12}\right|_{2} \zeta_{2}+\left.\chi_{12}\right|_{3} \zeta_{3}=\left.\chi_{12}\right|_{1}\left(\zeta_{1}+\frac{1}{2} \zeta_{3}\right)+\left.\chi_{12}\right|_{2}\left(\zeta_{2}+\frac{1}{2} \zeta_{3}\right) .
$$

It is readily verified that under this rule the natural curvature $\chi_{12}$ is constant over lines parallel to the triangle median that passes through node 3 . Formulas for the other curvatures follow by cyclic permutation, from which we construct the matrix relation

$$
\begin{aligned}
\left\{\begin{array}{l}
\chi_{12} \\
\chi_{23} \\
\chi_{31}
\end{array}\right\}= & {\left[\begin{array}{cccccc}
\zeta_{1}+\frac{1}{2} \zeta_{3} & \zeta_{2}+\frac{1}{2} \zeta_{3} & 0 & 0 & 0 & 0 \\
0 & 0 & \zeta_{2}+\frac{1}{2} \zeta_{1} & \zeta_{3}+\frac{1}{2} \zeta_{1} & 0 & 0 \\
0 & 0 & 0 & 0 & \zeta_{3}+\frac{1}{2} \zeta_{2} & \zeta_{1}+\frac{1}{2} \zeta_{2}
\end{array}\right] \mathrm{g} } \\
= & {\left[\begin{array}{cccccc}
6 \zeta_{12} & \left(3 \zeta_{21}-1\right) y_{21} & \left(3 \zeta_{12}+1\right) x_{21} & 6 \zeta_{21} & \left(3 \zeta_{21}+1\right) y_{21} & \left(3 \zeta_{12}-1\right) y_{21} \\
0 & 0 & 0 & 6 \zeta_{23} & \left(3 \zeta_{32}-1\right) y_{32} & \left(3 \zeta_{23}+1\right) x_{32} \\
6 \zeta_{13} & \left(3 \zeta_{13}+1\right) y_{13} & \left(3 \zeta_{31}-1\right) y_{13} & 0 & 0 & 0 \\
0 & 0 & 0 \\
& 6 \zeta_{32} & \left(3 \zeta_{32}+1\right) y_{32} & \left(3 \zeta_{23}-1\right) y_{32} \\
& 6 \zeta_{31} & \left(3 \zeta_{13}-1\right) y_{13} & \left(3 \zeta_{31}+1\right) x_{13}
\end{array}\right] \mathbf{v}, }
\end{aligned}
$$

in which $\zeta_{12}=\zeta_{1}-\zeta_{2}$, etc. In the notation of Box 3 ,

$$
\chi=A_{x a} g=A_{x a} Q v=B_{x a} v .
$$


where subscript $a$ identifies the "averaging" rule (37). Since the natural curvatures vary linearly over the triangle, their mean values are obtained by evaluating (39) at the centroid $\zeta_{1}=\zeta_{2}=\zeta_{3}=1 / 3$ :

$$
\overline{\boldsymbol{\chi}}=\left\{\begin{array}{l}
\bar{\chi}_{12} \\
\bar{\chi}_{23} \\
\bar{\chi}_{31}
\end{array}\right\}=\left[\begin{array}{ccccccccc}
0 & -y_{21} & x_{21} & 0 & y_{21} & -x_{21} & 0 & 0 & 0 \\
0 & 0 & 0 & 0 & -y_{32} & x_{32} & 0 & y_{32} & -x_{32} \\
0 & y_{13} & -x_{13} & 0 & 0 & 0 & 0 & -y_{13} & x_{13}
\end{array}\right] \mathbf{v}=\overline{\mathbf{B}}_{x^{2}} \mathbf{v} .
$$

Finally, the Cartesian curvatures are given by

$$
\kappa=\mathbf{T B}_{\chi a} \mathbf{v}=\mathbf{B}_{a} \mathbf{v}
$$

An explicit expression of these relations is easily obtained, but not required in what follows; however, that of the mean Cartesian curvatures $\overline{\boldsymbol{\kappa}}=\mathbf{T} \overline{\mathbf{B}}_{x a} \mathbf{v}=\overline{\mathbf{B}}_{a} \mathbf{v}$ (a relation valid because $\mathbf{T}$ is constant over the triangle) is enlightening:

$$
\bar{\kappa}=\left\{\begin{array}{c}
\bar{\kappa}_{x x} \\
\bar{\kappa}_{y y} \\
2 \bar{\kappa}_{x y}
\end{array}\right\}=\frac{1}{2 A}\left[\begin{array}{ccccccccc}
0 & 0 & y_{32} & 0 & 0 & y_{13} & 0 & 0 & y_{21} \\
0 & x_{32} & 0 & 0 & x_{13} & 0 & 0 & x_{21} & 0 \\
0 & y_{23} & x_{23} & 0 & y_{31} & x_{31} & 0 & y_{12} & x_{12}
\end{array}\right] \mathbf{v}=\overline{\mathbf{B}}_{\mathrm{c}} \mathbf{v}
$$

\subsection{The Projection Rule}

To each corner $k$ assign the natural curvature $\chi_{i j}$ of its projection onto the opposite side. This results in $\chi_{i j}$ being constant along lines normal to side $i j$. For equilateral triangles this agrees with the averaging rule, but not otherwise. The underlying motivation is to make the element insensitive to bad aspect ratios in cylindrical bending along side directions.

To illustrate the application of this rule consider side 1-2. For node 3 take

$$
\left.\chi_{12}\right|_{3}=\left.\frac{\partial^{2} w}{\partial \mu_{12}^{2}}\right|_{3}=\left.\lambda_{12} \chi_{12}\right|_{1}+\left.\lambda_{21} \chi_{12}\right|_{2}
$$

where $\lambda_{12}$ and $\lambda_{21}$ are defined in (23). Proceeding similarly along the other sides we construct the matrix relation

$$
\left\{\begin{array}{c}
\chi_{12} \\
\chi_{23} \\
\chi_{31}
\end{array}\right\}=\left[\begin{array}{cccccc}
\zeta_{1}+\lambda_{12} \zeta_{3} & \zeta_{2}+\lambda_{21} \zeta_{3} & 0 & 0 & 0 & 0 \\
0 & 0 & \zeta_{2}+\lambda_{23} \zeta_{1} & \zeta_{3}+\lambda_{32} \zeta_{1} & 0 & 0 \\
0 & 0 & 0 & 0 & \zeta_{3}+\lambda_{31} \zeta_{2} & \zeta_{1}+\lambda_{13} \zeta_{2}
\end{array}\right] \mathrm{g},
$$

or

$$
\chi=A_{x p} g, \quad \kappa=\mathbf{T A} A_{x p} \mathbf{g} .
$$

where subscript $p$ identifies the "projection" rule. As in the preceding rule, since $\mathbf{T}$ is constant we can do the strain-splitting step of Box 3 directly on the natural curvatures by evaluating at the centroid:

$$
\begin{aligned}
\mathbf{A}_{x p} & =\left(\overline{\mathbf{A}}_{x p}+\mathbf{A}_{x d p}\right) \\
& =\left[\begin{array}{cccccc}
\frac{1}{3}\left(1+\lambda_{12}\right) & \frac{1}{3}\left(1+\lambda_{21}\right) & 0 & 0 & 0 & 0 \\
0 & 0 & \frac{1}{3}\left(1+\lambda_{23}\right) & \frac{1}{3}\left(1+\lambda_{32}\right) & 0 & 0 \\
0 & 0 & 0 & 0 & \frac{1}{3}\left(1+\lambda_{31}\right) & \frac{1}{3}\left(1+\lambda_{13}\right)
\end{array}\right] \\
& +\left[\begin{array}{cccccc}
\zeta_{10}+\lambda_{12} \zeta_{30} & \zeta_{20}+\lambda_{21} \zeta_{30} & 0 & 0 & 0 & 0 \\
0 & 0 & \zeta_{20}+\lambda_{23} \zeta_{10} & \zeta_{30}+\lambda_{32} \zeta_{10} & 0 & 0 \\
0 & 0 & 0 & 0 & \zeta_{30}+\lambda_{31} \zeta_{20} & \zeta_{10}+\lambda_{13} \zeta_{20}
\end{array}\right] .
\end{aligned}
$$

in which $\zeta_{i 0}=\zeta_{i}-\frac{1}{3}$. Then

$$
\mathbf{B}_{p}=\mathbf{T} \mathbf{A}_{X p} \mathbf{Q}=\mathbf{T}\left(\overline{\mathbf{A}}_{x p}+\mathbf{A}_{d p}\right) \mathbf{Q}=\overline{\mathbf{B}}_{p}+\mathbf{B}_{d p} .
$$

The explicit expression of these matrices is not revealing and for the construction of the stiffness matrix presented in Appendix B it is better to leave (48) in product form. If all $\lambda$ coefficients are $\frac{1}{2}$, which happens for the equilateral triangle, the expressions reduce to those of the averaging rule. 


\subsection{The 'Sliding Beam' Rule}

This is a refinement of the average-curvature rule. Consider a fictitious beam parallel to side $i-j$ sliding towards corner $k$. The end displacements and rotation of this beam are obtained by interpolating $w$ cubically, $\theta_{n}$ quadratically, and $\theta_{t}$ linearly, along sides $i-k$ and $j-k$. Compute the mean natural curvature of this beam and assign to node $k$ the limit as the beam reaches that corner.

The required calculations can be simplified if we observe that the mean curvature of the sliding beam varies linearly as it moves from $i-j$, where it coincides with (41), to corner $k$. At one third of the way this mean is the natural centroidal curvature, which can then be readily extrapolated to $k$. These centroidal curvatures are given by $\bar{\chi}=\overline{\mathbf{B}}_{x s} \mathbf{v}$, where subscript $s$ identifies the 'sliding' rule. A symbolic calculation yields the explicit form

$$
\overline{\mathbf{B}}_{\chi s}^{T}=\left[\begin{array}{ccc}
2 \lambda_{13} & -2\left(\lambda_{21}+\lambda_{31}\right) & 2 \lambda_{12} \\
a_{2} c_{13} & a_{3} c_{21}+a_{2} c_{13} & a_{3} c_{21} \\
a_{2} s_{13} & a_{3} s_{21}+a_{2} s_{13} & a_{3} s_{21} \\
2 \lambda_{23} & 2 \lambda_{21} & -2\left(\lambda_{12}+\lambda_{32}\right) \\
a_{1} c_{32} & a_{3} c_{21} & a_{1} c_{32}+a_{3} c_{21} \\
a_{1} s_{32} & a_{3} s_{21} & a_{1} s_{32}+a_{3} s_{21} \\
-2\left(\lambda_{13}+\lambda_{23}\right) & 2 \lambda_{31} & 2 \lambda_{32} \\
a_{2} c_{13}+a_{1} c_{32} & a_{2} c_{13} & a_{1} c_{32} \\
a_{2} s_{13}+a_{1} s_{32} & a_{2} s_{13} & a_{1} s_{32}
\end{array}\right]
$$

where $a_{i}, c_{i j}$ and $s_{i j}$ are defined in Eqs. (23). Extrapolating to the opposite corners and interpolating over the triangle we get $\boldsymbol{\chi}=\mathbf{B}_{\chi}, \mathbf{v}$, with

$$
\mathbf{B}_{\chi s}^{T}=\left[\begin{array}{ccc}
6\left(-\zeta_{1}+\zeta_{2}+\lambda_{13} \zeta_{3}\right) & -6\left(\lambda_{21}+\lambda_{31}\right) \zeta_{1} & 6\left(\zeta_{3}-\zeta_{1}+\lambda_{12} \zeta_{2}\right) \\
2 y_{21}\left(1-3 \zeta_{1}\right)+3 a_{2} c_{13} \zeta_{3} & \left(3 a_{3} c_{21}+3 a_{2} c_{13}\right) \zeta_{1} & 2 y_{13}\left(3 \zeta_{1}-1\right)+3 a_{3} c_{21} \zeta_{2} \\
2 x_{21}\left(3 \zeta_{1}-1\right)+3 a_{2} s_{13} \zeta_{3} & \left(3 a_{3} s_{21}+3 a_{2} s_{13}\right) \zeta_{1} & 2 x_{13}\left(1-3 \zeta_{1}\right)+3 a_{3} s_{21} \zeta_{2} \\
6\left(\zeta_{1}-\zeta_{2}+\lambda_{23} \zeta_{3}\right) & 6\left(-\zeta_{2}+\zeta_{3}+\lambda_{21} \zeta_{1}\right) & -6\left(\lambda_{12}+\lambda_{32}\right) \zeta_{2} \\
2 y_{21}\left(3 \zeta_{2}-1\right)+3 a_{1} c_{32} \zeta_{3} & 2 y_{32}\left(1-3 \zeta_{2}\right)+3 a_{3} c_{21} \zeta_{1} & \left(3 a_{1} c_{32}+3 a_{3} c_{21}\right) \zeta_{2} \\
2 x_{21}\left(1-3 \zeta_{2}\right)+3 a_{1} s_{32} \zeta_{3} & 2 x_{32}\left(3 \zeta_{2}-1\right)+3 a_{3} s_{21} \zeta_{1} & \left(3 a_{1} s_{32}+3 a_{3} s_{21}\right) \zeta_{2} \\
-6\left(\lambda_{23}+\lambda_{13}\right) \zeta_{3} & 6\left(\zeta_{2}-\zeta_{3}+\lambda_{31} \zeta_{1}\right) & 6\left(-\zeta_{3}+\zeta_{1}+\lambda_{32} \zeta_{2}\right) \\
\left(3 a_{2} c_{13}+3 a_{1} c_{32}\right) \zeta_{3} & 2 y_{32}\left(3 \zeta_{3}-1\right)+3 a_{2} c_{13} \zeta_{1} & 2 y_{13}\left(1-3 \zeta_{3}\right)+3 a_{1} c_{32} \zeta_{2} \\
\left(3 a_{2} s_{13}+3 a_{1} s_{32}\right) \zeta_{3} & 2 x_{32}\left(1-3 \zeta_{3}\right)+3 a_{2} s_{13} \zeta_{1} & 2 x_{13}\left(3 \zeta_{3}-1\right)+3 a_{1} s_{32} \zeta_{2}
\end{array}\right] .
$$

It should be noted that $\mathbf{A} X$ and $\mathbf{Q}$ are inextricably enmeshed in the above formula and cannot be easily separated. Premultiplication by $T$ yields $\kappa=\mathbf{B}, \mathbf{v}$. Evaluation of $B$, at the centroid yields $\bar{B},=L_{q}^{T} / A$, where $\mathbf{L}_{q}^{T}=A \mathbf{T} \overline{\mathbf{B}}_{x}$, is the force lumping matrix given in Eq. (56).

$A$ variation in the sliding-beam theme would consist of interpolating the normal rotation $\theta_{n}$ along $i-k$ and $j-k$ linearly rather than quadratically. This scheme turns out to be identical, however, to the average curvature rule and thus it provides nothing new.

\subsection{The Six Beam Lattice Rule}

In addition to the sides, consider three fictitious beams along the triangle medians. Determine the displacements and rotations at the triangle midpoints by the same interpolation procedure as in the sliding beam rule. The linear curvatures along the medians are thus readily computed. At each triangle corner we now know the curvatures in three directions: the two sides and the median. Consequently we can transform to $x-y$ curvatures and interpolate these linearly over the element. This apparently new model gives. however, identical results to the projection rule, a result that can be a posteriori justified by geometric reasoning. Consequently this scheme will not be pursued further. 


\subsection{The ANS Elements}

Three ANS elements based on the previous interpolation rules may be constructed by following the prescription of Box 4. The stiffness matrices are identified as $\mathbf{K}_{a}, \mathbf{K}_{p}$, and $\mathbf{K}_{s}$, for averaging, projection, and sliding-beam, respectively. The following properties hold for these elements.

Patch Test. Assuming that the element has constant thickness and material properties, $\mathbf{K}_{a}$ and $K$, pass the individual element test, but $\mathbf{K}_{p}$ does not. This claim can be analytically confirmed by applying the criterion of Eqs. (16)-(17), and noting that $\overline{\mathbf{B}}_{a}=\mathbf{L}_{l}^{T} / A$ and $\overline{\mathbf{B}},=\mathbf{L}_{q}^{T} / A$, where $\mathbf{L}_{l}$ and $\mathbf{L}_{q}$ are the force lumping matrices derived in Section 4.

Equivalence with DKT. K, turns out to be identical to the stiffness matrix of the Discrete Kirchhoff Triangle (DKT) element, which was originally constructed in a completely different way $[3,4]$ that involves assumed rotation fields. Thus DKT is an ANS element, and also (because of the equivalence noted below) an ANDES element. This equivalence provides the first variational justification of DKT, as well as the proof that DKT passes the patch test without any numerical verification.

ANS/ANDES Equivalence. If the basic stiffness matrices $\mathbf{K}_{b l}$ and $\mathbf{K}_{b q}$ derived in Section 4.1 are used in conjunction with the averaging and sliding-beam rules, and $\alpha=1$, the ANDES formulation yields the same results as ANS if the element has constant thickness and material properties. (If the element has variable thickness, or the material properties vary, the equivalence does not hold.) The ANDES formulation used with the projection rule yields two elements, called ALR and AQR in the sequel, which differ in their basic stiffnesses. Both of these elements pass the patch test and are not equivalent to the ANS formulation.

\section{STIFFNESS MATRIX COMPUTATION}

\subsection{The Basic Stiffness}

As explained on Box 2, the basic stiffness is obtained by constructing the lumping matrix $L$. In our case this is a $9 \times 3$ matrix that "lumps" an internal constant bending-moment field $\left(\bar{m}_{x x}, \bar{m}_{y y}, \bar{m}_{x y}\right)$ to node forces $\overline{\mathbf{f}}$ conjugate to $\mathbf{v}$.

On each element side, the constant moment field produces boundary moments $\bar{m}_{n n}$ and $\bar{m}_{n t}$ referred to a local edge coordinate system $n, t$ (see Figure 3):

$$
\left\{\begin{array}{l}
\bar{m}_{n n} \\
\bar{m}_{n t}
\end{array}\right\}_{i j}=\left[\begin{array}{ccc}
s_{i j}^{2} & c_{i j}^{2} & -2 s_{i j} c_{i j} \\
s_{i j} c_{i j} & -s_{i j} c_{i j} & s_{i j}^{2}-c_{i j}^{2}
\end{array}\right]\left\{\begin{array}{l}
\bar{m}_{x x} \\
\bar{m}_{y y} \\
\bar{m}_{x y}
\end{array}\right\}
$$

The boundary motions $\mathrm{d}$ conjugate to $m_{n n}$ and $m_{n t}$ are $\partial w / \partial n=-\theta_{t}$ and $\partial w / \partial t=\theta_{n}$ (see Figure 3). Given the degree of freedom configuration (25), the normal slope $\partial w / \partial n=-\theta_{t}$ along side $i-j$ can at most vary linearly (it could be also taken as constant and equal to $\frac{1}{2}\left(\theta_{t i}+\theta_{t j}\right)$ but the results are the same as for a linear variation).

For the tangential slope (the rotation about the normal) $\partial w / \partial t=\theta_{n}$ there are three options: constant, linear and quadratic variation. But a constant $\theta_{n}=\left(w_{j}-w_{i}\right) / \ell_{i j}$ turns out to be equivalent to the quadratic variation and a constant $\theta_{n}=\frac{1}{2}\left(\theta_{n i}+\theta_{n j}\right)$ equivalent to the linear variation. Consequently only the linear and quadratic cases need to be examined.

Linear Normal Rotation. The variation of $\theta_{t}$ and $\theta_{n}$ along each side is linear:

$$
\left\{\begin{array}{l}
\theta_{t} \\
\theta_{n}
\end{array}\right\}_{i j}=\frac{1}{2}\left[\begin{array}{cccccc}
0 & 1-\xi & 0 & 0 & 1+\xi & 0 \\
0 & 0 & 1-\xi & 0 & 0 & 1+\xi
\end{array}\right]\left\{\begin{array}{c}
w_{i} \\
\theta_{t i} \\
\theta_{n i} \\
w_{j j} \\
\theta_{t j} \\
\theta_{n j}
\end{array}\right\}
$$


where $\xi \equiv \xi_{i j}$. Under this assumption one obtains [13]

$$
\mathbf{L}_{l}^{T}=\frac{1}{2}\left[\begin{array}{ccccccccc}
0 & 0 & y_{32} & 0 & 0 & y_{13} & 0 & 0 & y_{21} \\
0 & x_{32} & 0 & 0 & x_{13} & 0 & 0 & x_{21} & 0 \\
0 & y_{23} & x_{23} & 0 & y_{31} & x_{31} & 0 & y_{12} & x_{12}
\end{array}\right]
$$

where superscript $l$ stands for "linear $\theta_{n}$." The corresponding basic stiffness is

$$
\mathbf{K}_{b l}=A^{-1} \mathbf{L}_{l} \mathbf{D L} \mathbf{L}_{l}^{T}
$$

where $D$ is the Cartesian moment-curvature constitutive matrix resulting from the integration of $E$ through the plate thickness. This matrix been used as component of the free formulation (FF) element presented in Ref. [13].

Quadratic Normal Rotation. A quadratic variation of $\theta_{n}$ can be accomodated in conjunction with the cubic variation of $w$ along the side:

$$
\left\{\begin{array}{c}
\theta_{t} \\
\theta_{n}
\end{array}\right\}_{i j}=\frac{1}{2}\left[\begin{array}{cccccc}
0 & 1-\xi & 0 & 0 & 1+\xi & 0 \\
3\left(\xi^{2}-1\right) / \ell & 0 & \frac{1}{2}(3 \xi+1)(\xi-1) & 3\left(\xi^{2}-1\right) / \ell & 0 & \frac{1}{2}(3 \xi-1)(\xi+1)
\end{array}\right]\left\{\begin{array}{l}
w_{i} \\
\theta_{t i} \\
\theta_{n i} \\
w_{j} \\
\theta_{t j} \\
\theta_{n j}
\end{array}\right\}
$$

where $\xi \equiv \xi_{i j}$ and $\ell \equiv \ell_{i j}$. Then the resulting lumping matrix can be presented as

$$
\mathbf{L}_{q}=\left[\begin{array}{ccc}
-c_{12} s_{12}+c_{31} s_{31} & -c_{31} s_{31}+c_{12} s_{12} & \left(s_{31}^{2}-c_{31}^{2}\right)-\left(s_{12}^{2}-c_{12}^{2}\right) \\
\frac{1}{2}\left(s_{12}^{2} x_{12}+s_{31}^{2} x_{31}\right) & \frac{1}{2}\left(c_{12}^{2} x_{12}+c_{31}^{2} x_{31}\right) & c_{12}^{2} y_{21}+c_{31}^{2} y_{13} \\
-\frac{1}{2}\left(s_{12}^{2} y_{21}+s_{31}^{2} y_{13}\right) & -\frac{1}{2}\left(c_{12}^{2} y_{21}+c_{31}^{2} y_{13}\right) & -s_{12}^{2} x_{12}-s_{31}^{2} x_{31} \\
-c_{23} s_{23}+c_{12} s_{12} & -c_{12} s_{12}+c_{23} s_{23} & \left(s_{12}^{2}-c_{12}^{2}\right)-\left(s_{23}^{2}-c_{23}^{2}\right) \\
\frac{1}{2}\left(s_{12}^{2} x_{12}+s_{23}^{2} x_{23}\right) & \frac{1}{2}\left(c_{12}^{2} x_{12}+c_{23}^{2} x_{23}\right) & c_{12}^{2} y_{21}+c_{23}^{2} y_{32} \\
-\frac{1}{2}\left(s_{12}^{2} y_{21}+s_{23}^{2} y_{32}\right) & -\frac{1}{2}\left(c_{12}^{2} y_{21}+c_{23}^{2} y_{23}\right) & -s_{12}^{2} x_{12}-s_{23}^{2} x_{23} \\
-c_{31} s_{31}+c_{23} s_{23} & -c_{23} s_{23}+c_{31} s_{31} & \left(s_{23}^{2}-c_{23}^{2}\right)-\left(s_{31}^{2}-c_{31}^{2}\right) \\
\frac{1}{2}\left(s_{23}^{2} x_{23}+s_{31}^{2} x_{31}\right) & \frac{1}{2}\left(c_{23}^{2} x_{23}+c_{31}^{2} x_{31}\right) & c_{23}^{2} y_{32}+c_{31}^{2} y_{13} \\
-\frac{1}{2}\left(s_{23}^{2} y_{32}+s_{31}^{2} y_{13}\right) & -\frac{1}{2}\left(c_{23}^{2} y_{32}+c_{31}^{2} y_{13}\right) & -s_{23}^{2} x_{23}-s_{31}^{2} x_{31}
\end{array}\right] .
$$

The corresponding basic stiffness matrix is denoted by

$$
\mathbf{K}_{b q}=A^{-1} \mathbf{L}_{q} \mathbf{D L}_{q}^{T} \text {. }
$$

\subsection{The Higher Order Stiffness}

The higher order stiffness for the ANDES elements described in Section 3 is

$$
\mathbf{K}_{h x}=\alpha \mathbf{Q}^{T} \mathbf{K}_{d x} \mathbf{Q}=\alpha \mathbf{Q}^{T}\left[\int_{A} \mathbf{A}_{d x}^{T} \mathbf{D} \mathbf{A}_{d x} d A\right] \mathbf{Q}=\alpha \int_{A} \mathbf{B}_{d x}^{T} \mathbf{D} \mathbf{B}_{d x} d A
$$

where $x=a, p, s$ for the average, projection and sliding-beam rules, respectively. (The last expression is appropriate when $\mathbf{B}_{d x}$ is not easily factored into $\mathbf{A}_{d x} \mathbf{Q}$, as in the sliding-beam rule.) Since $\mathbf{A}_{d x}$ varies linearly, if $\mathrm{D}$ is constant we could numerically integrate $\mathbf{K}_{d x}$ in (58) exactly with a three point Gauss rule; for example the three-midpoint formula. But as the element stiffness formation time is dominated by these calculations it is of interest to derive $K_{h}$ in closed form. This is done in Appendix B for $\mathbf{K}_{h p}$. which from the numerical experiments appears to be the best performer. 


\section{NUMERICAL EXPERIMENTS: GENERAL DESCRIPTION}

An extensive set of numerical experiments has been run to assess the performance of the new ANDES elements based on the projection rule (ALR and AQR) and to compare them with other existing highperformance elements. Table 2 lists the tests, material properties and some relevant geometrical properties, whereas Table 3 lists elements, loading and mesh identifiers.

An inspection of the element identifiers in Table 3 displays two important points: the difference in the results obtained with AQR and ALR can be attributed to their basic stiffness, whereas differences between $A Q R$ and DKT can be attributed to their higher order stiffness. With these facts in mind, we conducted first a set of distortion tests so that the less distortion sensitive combinations can be identified. Then, the best performers are submitted to a set of representative thin-plate bending problems in linear elasticity.

The scaling $\alpha=1.5$ for ALR and $\alpha=1.0$ for AQR have been chosen to obtain energy balance in some simple cylindrical bending tests. No further adjustment of these parameters was made. In the distortion tests we included the results obtained with the free formulation (FF) element presented in [13], since that reference did not report such tests.

Whenever the simply supported condition appears it implies that only the transverse displacement $w$ is restrained. It is equivalent to the SS1 condition described in Hughes' textbook [21].

For tests involving an uniform distributed load $q$, two node-force computation schemes are usually reported:

1. Triangular lumping (TL), in which one third of the load $q A$ is assigned to each triangle corners, and nodal moments are set to zero.

$$
\mathbf{f}^{T}=\frac{q A}{3}\left[\begin{array}{lllllllll}
1 & 0 & 0 & 1 & 0 & 0 & 1 & 0 & 0
\end{array}\right] .
$$

2. Consistent lumping (CL), in which the element node force vector is

$$
\mathbf{f}^{T}=\frac{q A}{3}\left[\begin{array}{llllll}
1 & \frac{y_{31}+y_{21}}{8} & \frac{x_{13}+x_{12}}{8} & 1 & \frac{y_{23}+y_{32}}{8} & \ldots
\end{array}\right]
$$

This lumping was obtained using the transverse displacement $w$ of the FF element in [13]. It is used for the ANS and ANDES elements as a matter of expediency, since for such elements a unique transverse displacement does not exist.

Inasmuch as the present elements pass the linear patch test by virtue of their construction, no validation experiments along these lines are necessary once the elements are correctly programmed.

\section{DISTORTION TESTS}

\subsection{Simply Supported Square Plate under Central Load}

This test was proposed by Kang [23]. The use of a coarse mesh exacerbates the distortion effect when far from of the converged solution. (In a fine mesh the distortion effect would be diluted.) The mesh and distortion parameter are shown in Figure 4. When the distortion parameter $a$ approaches 2.5 the mesh converges to a four element cross-diagonal mesh. Results are reported as a percentage of the deterioration with respect to the undistorted mesh.

The results given in Table 4 show that AQR is superior in this test. FF and ALR are the worst for $a>2$. $\mathrm{DKT}$ and $\mathrm{AQR}$ display low deterioration rate from $a=2$ up to $a=2.49$, but DKT behaves poorly for $a<2$. 
Table 2. Key to Material and Geometrical Data

\begin{tabular}{ll} 
Test & Description \\
\hline Square plate & $\begin{array}{l}\text { Isotropic material } \nu=0, E=1 ; \text { thickness } t=1 \text {, plate span } a=10 ; \\
\text { load scaled so that center deflection } w_{c}=100\end{array}$ \\
Cantilever beam & $\begin{array}{l}\text { Isotropic material } \nu=0, E=1 ; \text { thickness } t=1 \text {; load scaled so } \\
\text { that center deflection } w_{c}=100\end{array}$ \\
Twisted ribbon & $\begin{array}{l}\text { Isotropic material } \nu=0.25, E=10^{7} ; \text { thickness } t=0.05 ; \text { transverse } \\
\text { load at tip so that } \mathbf{P}_{B}=-\mathbf{P}_{A}=1\end{array}$ \\
Rhombic cantilever & $\begin{array}{l}\text { Isotropic material } \nu=0.3, E=10.510^{6} ; \text { thickness } t=0.125 ; \\
\text { uniform transverse load } q=0.26066\end{array}$ \\
Rhombic plate & $\begin{array}{l}\text { Isotropic material } \nu=0.3, E=1 ; \text { thickness } t=1, \text { plate side } \\
a=100, \text { uniform transverse load } q \text { scaled so that } w_{c}=100\end{array}$
\end{tabular}

Table 3. Key to Element, Loading and Mesh Identifiers

\begin{tabular}{|ll|}
\hline Key & Explanation \\
\hline ALR & ANDES element $\mathbf{K}_{b l}+1.5 \mathbf{K}_{h p}$ \\
AQR & ANDES element $\mathbf{K}_{b q}+\mathbf{K}_{h p}$ \\
FF & FF element of [13] with 3-parameter scaling of $\mathbf{K}_{h}$ \\
DKT & ANS element $\mathbf{K}_{b q}+\mathbf{K}_{h,}$ : identical to DKT \\
\hline CL & Consistent lumping (59) of uniform load $q$ \\
TL & Triangular lumping (60) of uniform load $q$ \\
\hline SDC & In rhombic meshes, triangles obtained by splitting quadrilateral \\
& mesh units with short diagonal cuts \\
LDC & In rhombic meshes, triangles obtained by splitting quadrilateral \\
& mesh units with long diagonal cuts \\
\hline
\end{tabular}




\subsection{Cantilever Beam}

A cantilever beam with a transverse load at the tip was selected for this test. Two meshes shown in Figure 5 , $A$ and $B$, are used to observe the effect of the element orientation under a linear bending state. The results are reported in Table 5. Also shown in this table is the ratio of the computed tip deflection to the exact value $w_{\text {ex }}$ for zero distortion.

For mesh A, AQR is the best performer closely followed by DKT. FF and ALR behave poorly.

For mesh B FF is the best performer in terms of deterioration, followed by AQR, DKT and ALR. However it must be noted that FF and ALR recover only $77 \%$ of the exact solution. This is a serious drawback in elements supposedly capable of providing an appropriate response for linear bending. This shortcoming can be attributed to the basic stiffness $\mathbf{K}_{b /}$ which is the same for both elements. AQR and DKT recover almost $99 \%$ of the response for both meshes.

\subsection{Twisted Ribbon}

This test has been selected to assess the distortion effect under a field which combines bending and twisting. The test uses mesh $B$ of Figure 5. The results shown in Table 6 indicate that AQR and DKT are the least distortion sensitive elements for this problem. 

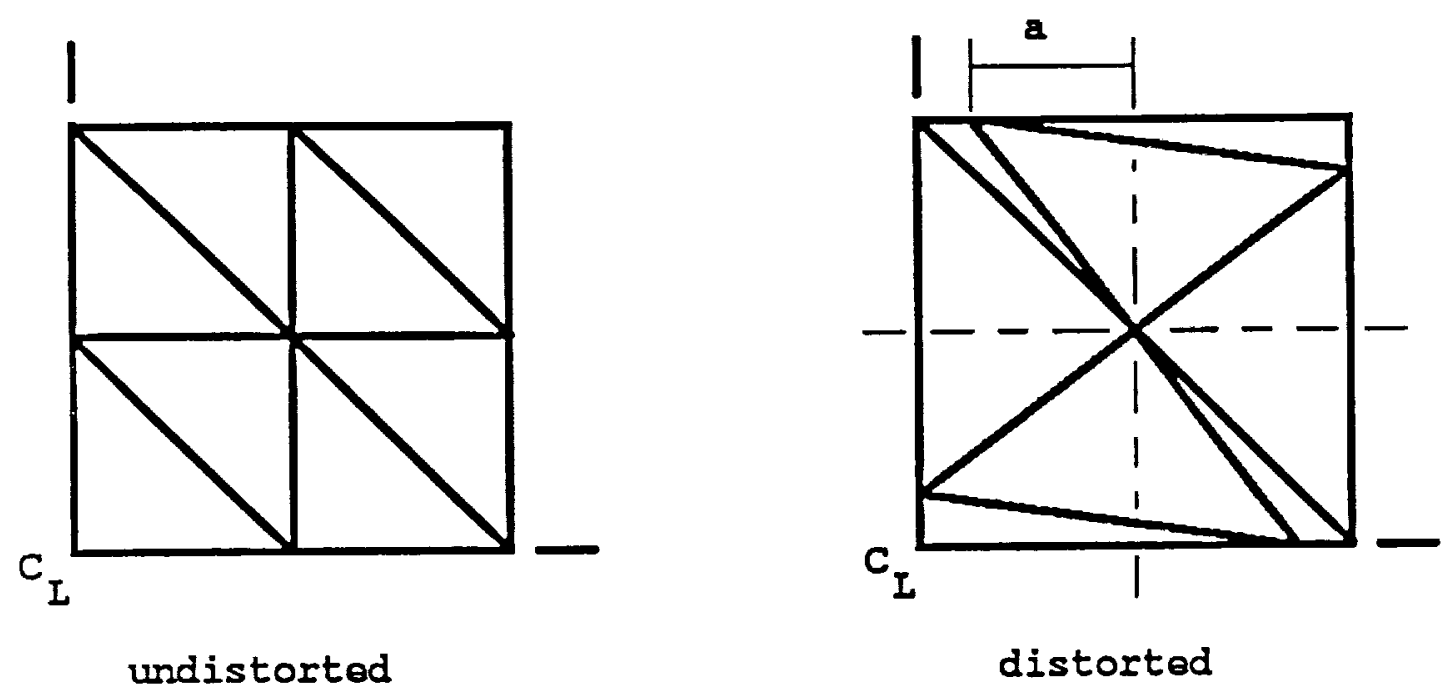

Figure 4. Square plate: mesh for distortion analysis

Table 4. Distortion Analysis: Centrally Loaded SS Square Plate: Percent Error of Center Deflection with Respect to Undistorted Mesh

\begin{tabular}{lrrrrr}
\hline $\begin{array}{l}\text { Element } \\
\text { type }\end{array}$ & 0.50 & 1.00 & 1.50 & 2.00 & 2.49 \\
\hline & & & & & \\
ALR & 0.83 & 2.65 & 5.05 & 7.88 & 10.38 \\
AQR & 0.17 & -0.14 & -1.59 & -3.29 & -4.40 \\
DKT & -0.95 & -3.46 & -6.29 & -8.06 & -8.42 \\
FF & 0.81 & 2.27 & 3.69 & 4.85 & -13.50 \\
\hline
\end{tabular}




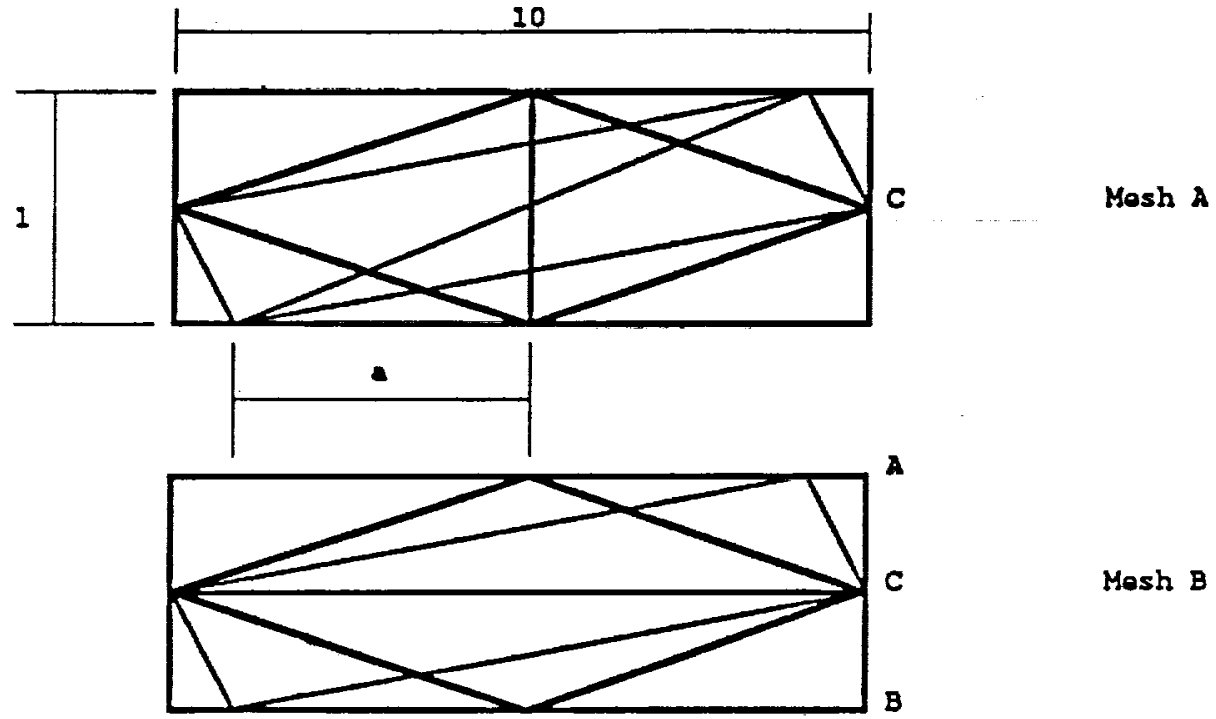

Figure 5. Distorted meshes for cantilever beam and twisted ribbon

Table 5. Distortion Analysis: Cantilever Beam

Percent Error at Node C with Respect to Undistorted Mesh

\begin{tabular}{llrrrr}
\hline Mesh & Element & \multicolumn{5}{c}{ Distortion parameter } & $w_{C} / w_{\text {Cex }}$ \\
& type & 1.00 & 3.00 & 4.90 & (no distortion) \\
\hline & & & & & \\
A & ALR & -10.70 & -19.80 & 8.40 & 1.031 \\
A & AQR & 0.15 & 0.10 & -2.05 & 0.995 \\
A & DKT & 0.20 & -0.59 & 3.41 & 0.982 \\
A & FF & -7.75 & -17.30 & -18.35 & 0.974 \\
\hline B & ALR & 0.20 & 3.00 & 45.90 & 0.764 \\
B & AQR & -0.10 & 0.40 & -2.85 & 0.995 \\
B & DKT & -0.13 & -1.09 & -3.49 & 0.979 \\
B & FF & -0.05 & -0.15 & 2.20 & 0.769 \\
\hline
\end{tabular}


Table 6. Distortion Analysis - Twisted Ribbon

Loss of Symmetry under Distortion (Mesh B)

\begin{tabular}{lcccc}
\hline $\begin{array}{l}\text { Element } \\
\text { type }\end{array}$ & Node & \multicolumn{4}{c}{ Distortion parameter } \\
& & 1.00 & 3.00 & 4.90 \\
\hline \multirow{2}{*}{ ALR } & A & 1.016 & 1.122 & 1.363 \\
& $\mathrm{~B}$ & 1.013 & 1.098 & 1.076 \\
AQR & $\mathrm{A}$ & 0.989 & 0.966 & 0.945 \\
& $\mathrm{~B}$ & 1.010 & 1.029 & 0.995 \\
DKT & $\mathrm{A}$ & 0.993 & 0.978 & 0.940 \\
& $\mathrm{~B}$ & 1.006 & 1.015 & 1.018 \\
FF & $\mathrm{A}$ & 0.983 & 0.933 & 0.789 \\
& $\mathrm{~B}$ & 0.994 & 0.877 & 0.877 \\
\hline
\end{tabular}

\section{CONVERGENCE STUDIES}

From the distortion test results, it can be concluded that elements whose basic stiffness is $\mathbf{K}_{b q}$ are less distortion sensitive. Consequently only results for the AQR and DKT elements are presented in the following studies.

\subsection{Square Plate}

In this analysis a square plate with either simply-supported or clamped edges is considered. Due to symmetry only one quarter of the plate is modeled. The two different mesh orientations, A and B, used in the analysis are illustrated in Figure 6 . The number of elements used is $2 N^{2}$, where $N$ is the number of side subdivisions.

For the cases involving a concentrated load, Figures 7 and 8 show that for both meshes AQR converges faster and is less sensitive to mesh orientation than DKT.

In the case of uniform loading with triangular lumping, Figures 9 and 10 , the convergence is uniform for all the meshes and elements. For the simply-supported condition all answers are within the $5 \%$ error limit for $N=4$. Clearly DKT converges faster in this case. For the clamped condition and $N=4$, DKT $(A)$ is outside the $5 \%$ error limit.

For consistent force lumping, the results shown in Table 7 indicate a dramatic improvement of AQR. DKT also improves in the sense that becomes less mesh sensitive and that all the results are within $5 \%$ error for $N=4$.

\subsection{Rhombic Cantilever}

The test involves a rhombic cantilevered plate subjected to uniform load. This problem was presented in [3] to test the DKT element with reference given to an experimental deflection result; however, no convergence analysis was performed. This has been done here taking into account the two possible mesh subdivision patterns, SDC and LDC, depicted in Figure 11. Triangular force lumping has been used.

The results are shown in Table 8 . For the LDC mesh DKT converges from above to an answer $4 \%$ below the experimental value quoted in [3]. On the other hand, AQR converges from below. For the SDC mesh 

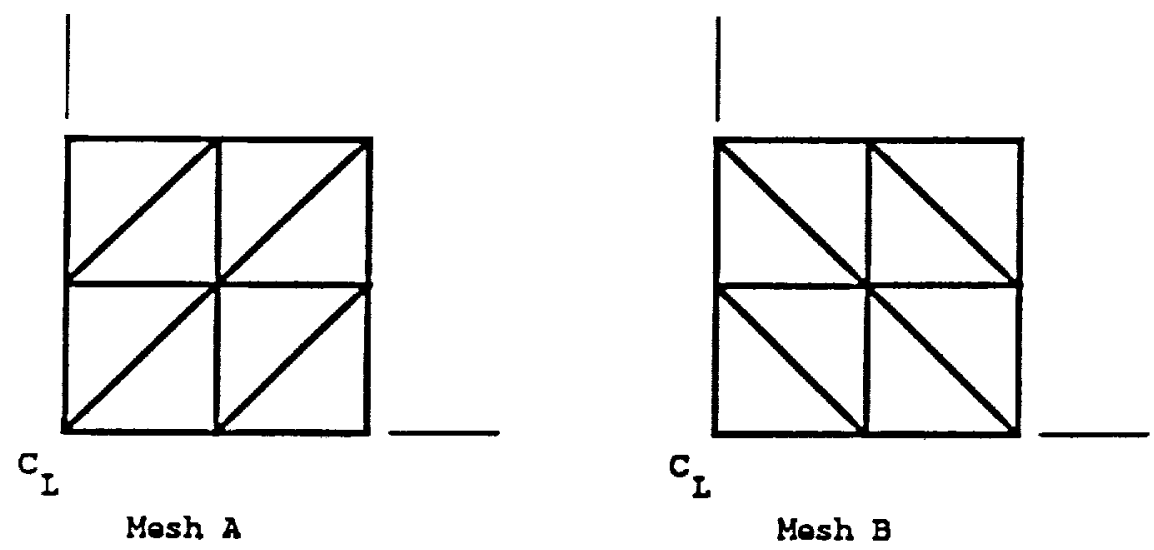

Figure 6. Meshes for square plate convergence studies

both elements behave identically and converge to a value $4 \%$ under the experimental one.

It is clear from these results that the experimental tip deflection given in [3] is in error by about $+4 \%$ with respect to the analytical value for the material properties quoted. The apparently small error for the $2 \times 2$ DKT/LDC mesh is thus fortuitous.

\subsection{Simply Supported Rhombic Plate}

This problem poses severe difficulties for ordinary finite element methods because of the presence of a singularity in the bending moments at the obtuse corner. A detailed description of this problem may be seen for example in [13]. The acute angle $\alpha=30^{\circ}$ was selected for the test. Again both SDC and LDC meshes were tried.

The results are shown in Table 9. For the SDC meshes AQR and DKT show slight difference and almost the same rate of convergence. For the LDC meshes DKT is too flexible whereas AQR converges faster. 


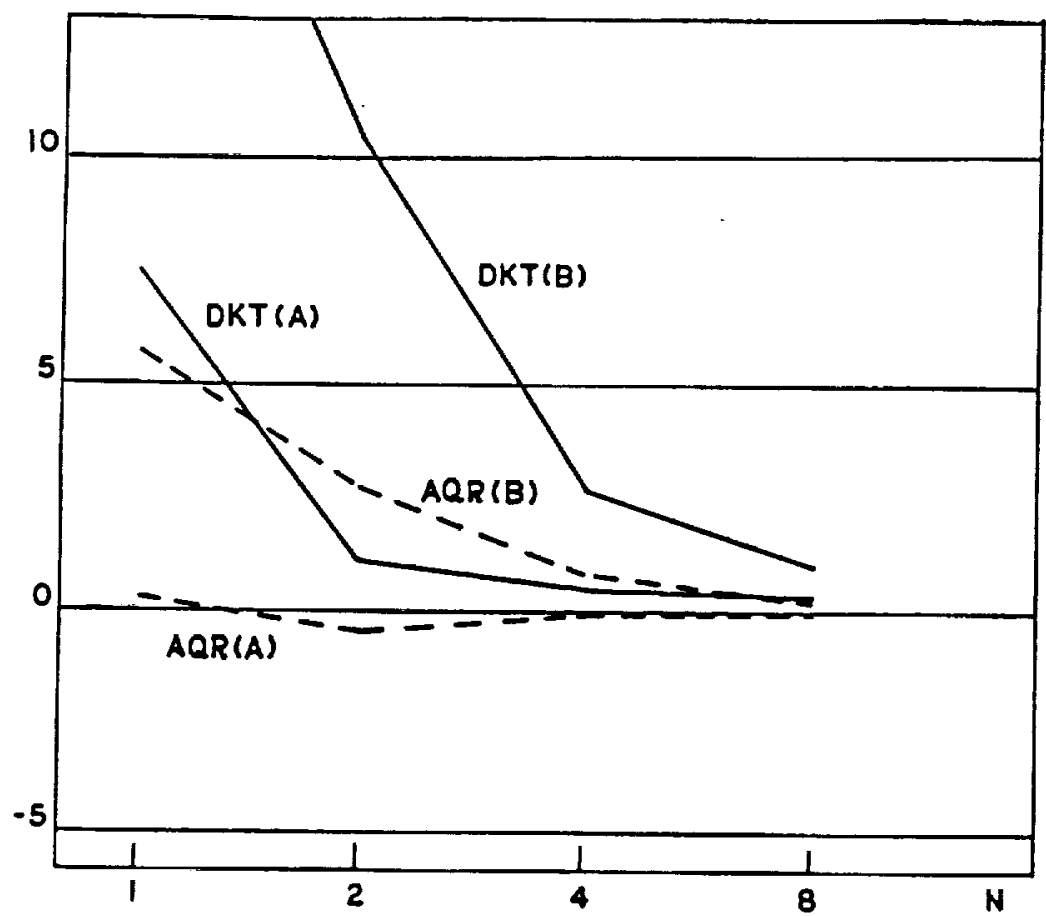

Figure 7. Central deflection of centrally loaded SS square plate



Figure 8. Central deflection of centrally loaded clamped square plate 




Figure 9. Central deflection of uniformly loaded SS square plate with TL force lumping

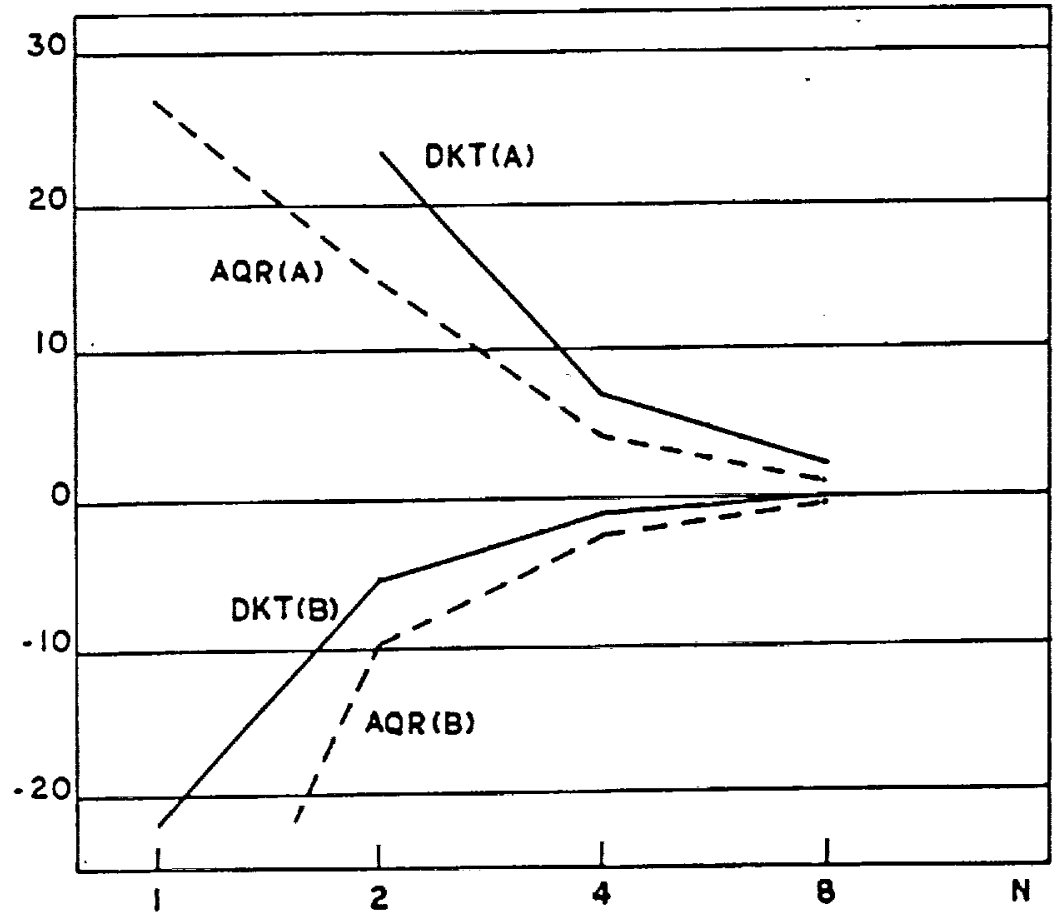

Figure 10. Central deflection of uniformly loaded clamped square plate with TL force lumping 
Table 7. Uniformly Loaded Square Plate with CL Force Lumping: Percent Error of Central Deflection

\begin{tabular}{lllrrrr}
\hline \multirow{2}{*}{$\begin{array}{l}\text { Support } \\
\end{array}$} & Element & Mesh & \multicolumn{5}{c}{ Mesh over quarter plate } \\
& type & type & $1 \times 1$ & $2 \times 2$ & $4 \times 4$ & $8 \times 8$ \\
\hline \multirow{2}{*}{ SS } & \multirow{2}{*}{ DKT } & A & 31.73 & 4.49 & 1.01 & 0.24 \\
& & B & 4.55 & 5.37 & 1.56 & 0.41 \\
& AQR & A & 16.28 & 2.20 & 0.47 & 0.11 \\
& & B & -1.55 & 2.30 & 0.74 & 0.20 \\
\hline \multirow{2}{*}{ Clamped } & DKT & A & 46.35 & 14.90 & 4.10 & 1.03 \\
& & B & -21.60 & 2.08 & 1.30 & 0.36 \\
& AQR & A & 26.65 & 8.26 & 1.87 & 0.44 \\
& & B & -41.20 & -3.22 & -0.28 & -0.05 \\
\hline
\end{tabular}

Table 8. Rhombic Cantilever: Percent Difference of Tip-A Deflection with Respect to Experimental Value of [3]

\begin{tabular}{llrrr}
\hline $\begin{array}{l}\text { Mesh } \\
\text { type }\end{array}$ & $\begin{array}{l}\text { Element } \\
\text { type }\end{array}$ & \multicolumn{4}{c}{ Subdivision of whole plate } \\
& & & $8 \times 4$ & $\mathbf{8} \times 8 \times 16$ \\
\hline \multirow{2}{*}{ LDC } & DKT & 2.3 & -3.7 & -4.0 \\
& AQR & -17.8 & -10.4 & -6.0 \\
\hline \multirow{3}{*}{ SDC } & DKT & -6.7 & -5.0 & -4.0 \\
& AQR & -6.3 & -5.0 & -4.0 \\
\hline
\end{tabular}




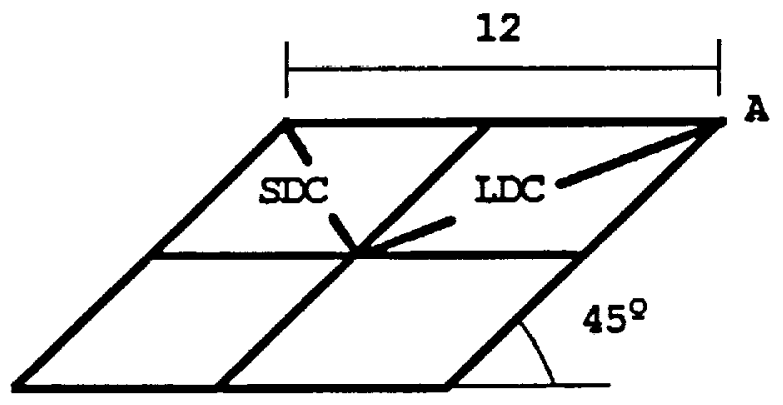

Figure 11. Rhombic cantilever: meshes for convergence studies

Table 0. Uniformly Loaded SS Rhombic Plate with TL Force Lumping: Percent Error in Center Deflection

\begin{tabular}{|c|c|c|c|c|}
\hline \multirow{2}{*}{$\begin{array}{l}\text { Mesh } \\
\text { type }\end{array}$} & \multirow{2}{*}{$\begin{array}{l}\text { Element } \\
\text { type }\end{array}$} & \multicolumn{3}{|c|}{ Subdivision of whole plate } \\
\hline & & $4 \times 4$ & $8 \times 8$ & $16 \times 16$ \\
\hline \multirow[t]{2}{*}{ SDC } & DKT & 11.05 & 4.07 & 2.86 \\
\hline & AQR & 13.86 & 4.56 & 2.89 \\
\hline \multirow[t]{2}{*}{ LDC } & DKT & 80.97 & 22.64 & 7.51 \\
\hline & AQR & 6.85 & -0.36 & -2.91 \\
\hline
\end{tabular}




\section{CONCLUSIONS}

The main conclusions of the present study can be summarized as follows.

1. The ANDES formulation represents a variant of the ANS formulation that merits serious study. The key advantages of ANDES over ANS are:

(a) a priori satisfaction of the patch test. Although this advantage is less clear for elements where ANS and ANDES coalesce for constant thickness and material properties, it reappears for more general cases.

(b) The separation of the higher order stiffness allows the application of a scaling parameter. Furthermore it opens the possibility for the energy-balanced combination with other formulations as per Eq. (2), although this possibility presently remains unexplored.

2. The study of plate bending elements shows that the widely used DKT element is both an ANS and ANDES element. This discovery provides a variational foundation hereto lacking and analytically proves (because of the ANDES connection) that DKT passes the patch test.

3. The numerical results clearly demonstrate that the choice of basic stiffness is of paramount importance in the behavior of elements based on the ANDES formulation. Of the two elements sharing the quadraticrotation basic stiffness, namely AQR and DKT, the former has excelled in geometric distortion tests and in convergence studies that involve concentrated forces. For other cases the performance of AQR and DKT is similar, and superior to those elements that use the linear-rotation basic stiffness.

The numerical experiments have not addressed questions of material sensitivity such as element performance for highly anisotropic and composite plates. This behavior, as well as the possibility of applying this technology to $C^{0}$ bending elements, is currently under investigation. 


\section{Acknowledgements}

The work of the first author has been supported by a fellowship from the Consejo Nacional de Investigaciones Cientificas y Técnicas (CONICET), Argentina. The work of the second author has been partly supported by NASA Lewis Research Center under Grant NAG 3-934 and by NSF under Grant ASC-8717773.

\section{References}

1. D. G. Ashwell, Strain elements, with applications to arches, rings and cylindrical shells, in Finite Elements for Thin Shells and Curved Members, ed. by D. G. Ashwell and R. H. Gallagher, Wiley-Interscience, London, 1976

2. K. J. Bathe and E. N. Dvorkin, A four-node plate bending element based on Mindlin/Reissner plate theory and a mixed interpolation, Int. J. Numer. Meth. Engrg., 21, 1985, pp. 367-383

3. J. L. Batoz, K. J. Bathe and L. W. Ho, A study of three-node triangular plate bending elements, Int. J. Numer. Meth. Engng, 15, 1980, pp. 1771-1812

4. J. L. Batoz, An explicit formulation for an efficient triangular plate-bending element, Int. J. Numer. Meths. Engrg., 18, pp. 1077-1089, 1982

5. G. P. Bazeley, Y. K. Cheung, B. M. Irons and O. C. Zienkiewicz, Triangular elements in plate bending: conforming and non-conforming solutions, Proceedings 1st Conference on Matrix Methods in Struclural Mechanics, AFFDL-TR-66-80, Air Force Institute of Technology, Wright-Patterson Air Force Base, Dayton, Ohio, 1966

6. P. G. Bergan and L. Hanssen, A new approach for deriving 'good' finite elements, MAFELAP II Conference, Brunel University, 1975, in The Mathemotics of Finite Elements and Applications - Volume $I I$, ed. by J. R. Whiteman, Academic Press, London, 1976

7. P. G. Bergan, Finite elements based on energy orthogonal functions, Int. J. Num. Meth. Engrg., 15, 1980, pp. 1141-1555

8. P. G. Bergan and M. K. Nygard, Finite elements with increased freedom in choosing shape functions, Int. J. Num. Meth. Engrg., 20, 1984, pp. 643-664

9. P. G. Bergan and C. A. Felippa, A triangular membrane element with rotational degrees of freedom, Computer Methods in Applied Mechanics \& Engineering, 50, 1985, pp. 25-69

10. P. G. Bergan and C. A. Felippa, Efficient implementation of a triangular membrane element with drilling Greedoms, Finite Element Handbook series, ed. by T. J. R. Hughes and E. Hinton, Pineridge Press, 1986. pp. 139-152

11. M. A. Crisfield, A four-noded thin plate element using shear constraints - a modified version of Lyons' element, Computer Methods in Applied Mechanics \& Engineering, 38, 1983, pp. 93-120

12. C. A. Felippa, Refined finite element analysis of linear and nonlinear two-dimensional structures. Ph. D. Dissertation, Department of Civil Engineering, University of California at Berkeley, Berkeley, Cal., 1966

13. C. A. Felippa and P. G. Bergan, A triangular plate bending element based on an energy-orthogonal free formulation, Computer Methods in Applied Mechanics \& Engineering, 61, 1987, pp. 129-160

14. C. A. Felippa, Parametrized multifield variational principles in elasticity: I. Mixed functionals. Communications in Applied Numerical Methods, 5, 1989, pp. 69-78

15. C. A. Felippa, Parametrized multifield variational principles in elasticity: II. Hybrid functionals and the free formulation, Communications in Applied Numerical Methods, 5, 1989, pp. 79-88

16. C. A. Felippa, The extended free formulation of finite elements in linear elasticity, Journal of Applied Mechanics, 56, 3, 1989, pp. 609-616 
17. C. A. Felippa and C. Militello, Variational formulation of high performance finite elements: parametrized variational principles, to appear in Computers $\mathscr{O}$ Structures

18. C. A. Felippa and C. Militello, Developments in variational methods for high performance plate and shell elements, in Analytical and Computational Models for Shells, CED Vol. 3, Eds. A. K. Noor, T. Bely tschko and J. C. Simo, The American Society of Mechanical Engineers, ASME, New York, 1989, pp. 191-216

19. H. C. Huang and E. Hinton, A new nine node degenerated shell element with enhanced membrane and shear interpolation, Int. J. Numer. Meth. Engrg., 22, 1986, pp. 73-92

20. T. J. R. Hughes and T.E. Tezduyar, Finite elements based upon Mindlin plate theory with particular reference to the four-node bilinear isoparametric element, Journal of Applied Mechanics, 48, 1981, pp. 587-596

21. T. J. R. Hughes, The Finite Element Method: Linear Static and Dynamic Finite Element Analysis, Prentice-Hall, Englewood Clifts, N. J., 1987

22. J. Jang and P. M. Pinsky, An assumed covariant strain based 9-node shell element, Int. J. Numer. Meth. Engrg., 24, 1987, pp. $2389-2411$

23. D. S. S. Kang, Hybrid stress finite element method, $\mathrm{Ph}$. D. Dissertation, Dept. of Aeronautics and Astronautics, Massachusetts Institute of Technology, 1986

24. R. H. MacNeal, Derivation of element stiffness matrices by assumed strain distribution, Nuclear Engrgr. Design, 70, 1978, pp. 3-12

25. D. S. Malkus and T. J. R. Hughes, Mixed finite element methods - reduced and selective integration techniques: a unification of concepts, Compuler Methods in Applied Mechanics and Engineering, 15, 1978, pp. 68-81

26. C. Militello and C. A. Felippa, A variational justification of the assumed natural strain formulation of finite elements: I. Variational principles, Computers and Structures, in press

27. C. Militello and C. A. Felippa, A variational justification of the assumed natural strain formulation of finite elements: II. The four node $C^{\circ}$ plate element, Computers and Structures, in press

28. J. T. Oden and J. N. Reddy, Variational Methods in Theoretical Mechanics, 2nd ed., Springer-Verlag, Berlin. 1983

29. K. C. Park and G. M. Stanley, A curved $C^{\circ}$ shell element based on assumed natural-coordinate strains, Journal of Applied Mechanics, 53, 1986, pp. 278-290

30. K. C. Park, An improved strain interpolation for curved $C^{\circ}$ elements, Int. J. Numer. Meth. Engrg., 22, 1986 , pp. 281-288

31. J. C. Simo and T. J. R. Hughes, On the variational foundations of assumed strain methods, Journal of Applied Mechanics, 53, 1986, pp. 51-54

32. M. J. Turner, R. W. Clough, H. C. Martin, and L. J. Topp, Stiffness and deflection analysis of complex structures. Journal of Aeronautical Sciences, 23, 1956, pp. 805-824

33. I. J. Willam, Finite element analysis of cellular structures, Ph. D. Dissertation, Dept. of Civil Engineering. University of California, Berkeley CA, 1969 


\section{Appendix A. SANITIZING INCOMPATIBLE ELEMENTS}

The stiffness-splitting technique summarized in Box 1 provides a systematic way for "sanitizing" existing nonconforming bending elements that do not pass the patch test. The technique amounts to the replacement of the basic stiffness. The main steps will be briefly outlined for the simplest such element: the BCIZ triangle proposed in 1965 by Bazeley et al [5]. The assumed transverse displacement is given explicitly in [12] as

$$
w=\left\{\begin{array}{c}
\zeta_{1}^{2}\left(3-2 \zeta_{1}\right)+2 \zeta_{1} \zeta_{2} \zeta_{3} \\
\zeta_{1}^{2}\left(y_{12} \zeta_{2}-y_{31} \zeta_{3}\right)+\widetilde{y}_{1} \zeta_{1} \zeta_{2} \zeta_{3} \\
\zeta_{1}^{2}\left(x_{21} \zeta_{2}-x_{13} \zeta_{3}\right)+\widetilde{x}_{1} \zeta_{1} \zeta_{2} \zeta_{3} \\
\zeta_{2}^{2}\left(3-2 \zeta_{2}\right)+2 \zeta_{1} \zeta_{2} \zeta_{3} \\
\zeta_{2}^{2}\left(y_{23} \zeta_{3}-y_{12} \zeta_{1}\right)+\widetilde{y}_{2} \zeta_{1} \zeta_{2} \zeta_{3} \\
\zeta_{2}^{2}\left(x_{32} \zeta_{3}-x_{21} \zeta_{1}\right)+\widetilde{x}_{2} \zeta_{1} \zeta_{2} \zeta_{3} \\
\zeta_{3}^{2}\left(3-2 \zeta_{3}\right)+2 \zeta_{1} \zeta_{2} \zeta_{3} \\
\zeta_{3}^{2}\left(y_{31} \zeta_{1}-y_{23} \zeta_{2}\right)+\widetilde{y}_{3} \zeta_{1} \zeta_{2} \zeta_{3} \\
\zeta_{3}^{2}\left(x_{13} \zeta_{1}-x_{32} \zeta_{2}\right)+\widetilde{x}_{3} \zeta_{1} \zeta_{2} \zeta_{3}
\end{array}\right\}^{T} \mathbf{v}
$$

where $\tilde{y}_{1}=y_{12}-y_{31}, \tilde{y}_{2}=y_{23}-y_{12}, \tilde{y}_{3}=y_{31}-y_{23}, \tilde{x}_{1}=x_{21}-x_{13}, \tilde{x}_{2}=x_{32}-x_{21}, \tilde{x}_{3}=x_{13}-x_{32}$. The strain-displacement matrix $B$ is obtained by double differentiation with respect to the triangular coordinates and application of (30):

$$
\kappa=\left\{\begin{array}{c}
\kappa_{x x} \\
\kappa_{y y} \\
2 \kappa_{x y}
\end{array}\right\}=\mathbf{W R v}=\mathbf{B v}=\left(B_{0}+B_{1} \zeta_{1}+B_{2} \zeta_{2}+B_{3} \zeta_{3}\right) \mathbf{v}
$$

in which $\mathbf{W}$ is given by (29), and

$$
\mathbf{R}^{T}=2\left[\begin{array}{cccccc}
3\left(1-\zeta_{1}\right) & 0 & 0 & \zeta_{3} & \zeta_{1} & \zeta_{2} \\
y_{12} \zeta_{2}-y_{31} \zeta_{3} & 0 & 0 & y_{12} \zeta_{1}+\frac{1}{2} \tilde{y}_{1} \zeta_{3} & \frac{1}{2} \tilde{y}_{1} \zeta_{1} & -y_{31} \zeta_{1}+\frac{1}{2} \tilde{y}_{1} \zeta_{2} \\
x_{12} \zeta_{2}-x_{31} \zeta_{3} & 0 & 0 & x_{21} \zeta_{1}+\frac{1}{2} \tilde{x}_{1} \zeta_{3} & \frac{1}{2} \tilde{x}_{1} \zeta_{1} & -x_{13} \zeta_{1}+\frac{1}{2} \tilde{x}_{1} \zeta_{2} \\
0 & 3\left(1-\zeta_{2}\right) & 0 & \zeta_{3} & \zeta_{1} & \zeta_{2} \\
0 & y_{23} \zeta_{3}-y_{12} \zeta_{1} & 0 & -y_{12} \zeta_{2}+\frac{1}{2} \tilde{y}_{2} \zeta_{3} & y_{23} \zeta_{2}+\frac{1}{2} \tilde{y}_{2} \zeta_{1} & \frac{1}{2} \tilde{y}_{2} \zeta_{2} \\
0 & x_{32} \zeta_{3}-x_{21} \zeta_{1} & 0 & -x_{21} \zeta_{2}+\frac{1}{2} \tilde{x}_{2} \zeta_{3} & x_{32} \zeta_{2}+\frac{1}{2} \tilde{x}_{2} \zeta_{1} & \frac{1}{2} \tilde{x}_{2} \zeta_{2} \\
0 & 0 & 3\left(1-\zeta_{3}\right) & \zeta_{3} & \zeta_{1} & \zeta_{2} \\
0 & 0 & y_{31} \zeta_{1}-y_{23} \zeta_{2} & \frac{1}{2} \tilde{y}_{3} \zeta_{3} & -y_{23} \zeta_{3}+\frac{1}{2} \tilde{y}_{3} \zeta_{2} & y_{31} \zeta_{3}+\frac{1}{2} \tilde{y}_{3} \zeta_{1} \\
0 & 0 & x_{13} \zeta_{1}-x_{32} \zeta_{2} & \frac{1}{2} \tilde{x}_{3} \zeta_{3} & -x_{32} \zeta_{3}+\frac{1}{2} \tilde{x}_{3} \zeta_{2} & x_{13} \zeta_{3}+\frac{1}{2} \tilde{x}_{3} \zeta_{1}
\end{array}\right] .
$$

Split the strain-displacement equations as

$$
\kappa=\bar{\kappa}+\kappa_{d}=\left(\overline{\mathbf{B}}+\mathbf{B}_{d}\right) \mathbf{v}
$$

where $\overline{\mathbf{B}}=\mathbf{B}_{0}+\frac{1}{3}\left(\mathbf{B}_{1}+\mathbf{B}_{2}+\mathbf{B}_{3}\right), \mathbf{B}_{d}=\mathbf{B}-\overline{\mathbf{B}}$. Then the "sanitized" stiffness matrix is

$$
\mathbf{K}=\mathbf{K}_{b}+\alpha \int_{A} \mathbf{B}_{d}^{T} \mathbf{D}_{b} \mathbf{B}_{d} d A
$$

where $K_{b}$ is one of the basic stiffness matrices derived in Section 4.1. The free formulation leads to the same result but in a less direct manner, because $w$ would have to be decomposed into rigid body, constant curvature and higher order states. Although the corrected element passes the patch test it is unlikely to be competitive with ANDES elements in distortion insensitivity as this property appears to depend on relaxing curvature compatibility conditions. 


\section{Appendix B. EXPLICIT REPRESENTATION OF HIGHER ORDER STIFFNESS}

To obtain an explicit representation of $\mathbf{K}_{h p}$, begin by defining

$$
\mathbf{C}=\mathbf{T}^{T} \mathbf{D T}=\left[\begin{array}{ccc}
C_{11} & C_{12} & C_{13} \\
& C_{22} & C_{23} \\
\text { symm } & & C_{23}
\end{array}\right],
$$

which can be interpreted as a constitutive matrix that relates the natural moments $\mathbf{T}^{T} \mathbf{m}$ to the natural curvatures $\chi$. Then

$$
\mathbf{K}_{d p}=\int_{A} \mathbf{A}_{d}^{T} \mathbf{C A}_{d} d A=\frac{A}{36}\left[\begin{array}{rrrrrr}
r_{11} & -r_{11} & r_{12} & -r_{12} & r_{13} & -r_{13} \\
& r_{11} & -r_{12} & r_{12} & -r_{13} & r_{13} \\
& & r_{22} & -r_{22} & r_{23} & -r_{23} \\
& & & r_{22} & -r_{23} & r_{23} \\
s y m m & & & & r_{33} & -r_{33} \\
& & & & & r_{33}
\end{array}\right]
$$

where $r_{i j}=\beta_{i j} C_{i j}$ for $i=1,2,3, j=1,2,3$, and

$$
\begin{gathered}
\beta_{11}=2\left(\lambda_{12}^{2}-\lambda_{12}+1\right), \quad \beta_{22}=2\left(\lambda_{23}^{2}-\lambda_{23}+1\right), \quad \beta_{33}=2\left(\lambda_{31}^{2}-\lambda_{31}+1\right), \\
\beta_{12}=\left(2-\lambda_{12}\right) \lambda_{23}-\lambda_{12}-1, \quad \beta_{23}=\left(2-\lambda_{23}\right) \lambda_{31}-\lambda_{23}-1, \quad \beta_{13}=\left(2-\lambda_{31}\right) \lambda_{12}-\lambda_{31}-1 .
\end{gathered}
$$

Carrying out the congruential transformation $K_{h p}=Q^{T} K_{d p} Q$ with MACSYMA yields

$$
\begin{aligned}
& K_{11}=4\left(r_{33}-r_{13}-r_{13}+r_{11}\right), \\
& K_{13}=2\left(\left(r_{13}-r_{11}\right) x_{21}+\left(r_{33}-r_{13}\right) x_{13}\right), \\
& K_{15}=2\left(\left(r_{12}-r_{23}\right) y_{32}+\left(r_{11}-r_{13}\right) y_{21}\right), \\
& K_{17}=4\left(-r_{33}+r_{23}+r_{13}-r_{12}\right), \\
& K_{19}=2\left(\left(r_{23}-r_{12}\right) x_{32}+\left(r_{33}-r_{13}\right) x_{13}\right), \\
& K_{23}=\left(-r_{11} x_{21}-r_{13} x_{13}\right) y_{21}+\left(-r_{13} x_{21}-r_{33} x_{13}\right) y_{13}, \\
& K_{25}=\left(r_{12} y_{21}+r_{23} y_{13}\right) y_{32}+r_{11} y_{21}^{2}+r_{13} y_{13} y_{21}, \\
& K_{27}=2\left(\left(r_{13}-r_{12}\right) y_{21}+\left(r_{33}-r_{23}\right) y_{13}\right), \\
& K_{29}=\left(-r_{12} x_{32}-r_{13} x_{13}\right) y_{21}+\left(-r_{23} x_{32}-r_{33} x_{13}\right) y_{13}, \\
& K_{34}=2\left(\left(r_{11}-r_{12}\right) x_{21}+\left(r_{13}-r_{23}\right) x_{13}\right), \\
& K_{36}=\left(r_{12} x_{21}+r_{23} x_{13}\right) x_{32}+r_{11} x_{21}^{2}+r_{13} x_{13} x_{21}, \\
& K_{38}=\left(-r_{12} x_{21}-r_{23} x_{13}\right) y_{32}+\left(-r_{13} x_{21}-r_{33} x_{13}\right) y_{13}, \\
& K_{44}=4\left(r_{22}-r_{12}-r_{12}+r_{11}\right), \\
& K_{46}=2\left(\left(r_{12}-r_{22}\right) x_{32}+\left(r_{11}-r_{12}\right) x_{21}\right), \\
& K_{48}=2\left(\left(r_{22}-r_{12}\right) y_{32}+\left(r_{23}-r_{13}\right) y_{13}\right), \\
& K_{55}=r_{22} y_{32}^{2}+2 r_{12} y_{21} y_{32}+r_{11} y_{21}^{2}, \\
& K_{57}=2\left(\left(r_{23}-r_{22}\right) y_{32}+\left(r_{13}-r_{12}\right) y_{21}\right), \\
& K_{59}=\left(-r_{22} x_{32}-r_{23} x_{13}\right) y_{32}+\left(-r_{12} x_{32}-r_{13} x_{13}\right) y_{21}, \\
& K_{67}=2\left(\left(r_{22}-r_{23}\right) x_{32}+\left(r_{12}-r_{13}\right) x_{21}\right), \\
& K_{69}=r_{22} x_{32}^{2}+\left(r_{12} x_{21}+r_{23} x_{13}\right) x_{32}+r_{13} x_{13} x_{21}, \\
& K_{78}=2\left(\left(r_{23}-r_{22}\right) y_{32}+\left(r_{33}-r_{23}\right) y_{13}\right), \\
& K_{68}=r_{22} y_{32}^{2}+2 r_{23} y_{13} y_{32}+r_{33} y_{13}^{2}, \\
& K_{99}=r_{22} x_{32}^{2}+2 r_{23} x_{13} x_{32}+r_{33} x_{13}^{2} .
\end{aligned}
$$$$
K_{12}=2\left(\left(r_{11}-r_{13}\right) y_{21}+\left(r_{13}-r_{33}\right) y_{13}\right)
$$$$
K_{14}=4\left(-r_{23}+r_{13}+r_{12}-r_{11}\right)
$$$$
K_{16}=2\left(\left(r_{23}-r_{12}\right) x_{32}+\left(r_{13}-r_{11}\right) x_{21}\right)
$$$$
K_{18}=2\left(\left(r_{12}-r_{23}\right) y_{32}+\left(r_{13}-r_{33}\right) y_{13}\right)
$$$$
K_{22}=r_{11} y_{21}^{2}+2 r_{13} y_{13} y_{21}+r_{33} y_{13}^{2}
$$$$
K_{24}=2\left(\left(r_{12}-r_{11}\right) y_{21}+\left(r_{23}-r_{13}\right) y_{13}\right)
$$$$
K_{26}=\left(-r_{12} x_{32}-r_{11} x_{21}\right) y_{21}+\left(-r_{23} x_{32}-r_{13} x_{21}\right) y_{13}
$$$$
K_{28}=\left(r_{12} y_{21}+r_{23} y_{13}\right) y_{32}+r_{13} y_{13} y_{21}+r_{33} y_{13}^{2}
$$$$
K_{33}=r_{11} x_{21}^{2}+2 r_{13} x_{13} x_{21}+r_{33} x_{13}^{2}
$$$$
K_{35}=\left(-r_{12} x_{21}-r_{23} x_{13}\right) y_{32}+\left(-r_{11} x_{21}-r_{13} x_{13}\right) y_{21}
$$$$
K_{37}=2\left(\left(r_{12}-r_{13}\right) x_{21}+\left(r_{23}-r_{33}\right) x_{13}\right)
$$$$
K_{39}=\left(r_{12} x_{21}+r_{23} x_{13}\right) x_{32}+r_{13} x_{13} x_{21}+r_{33} x_{13}^{2}
$$$$
K_{45}=2\left(\left(r_{22}-r_{12}\right) y_{32}+\left(r_{12}-r_{11}\right) y_{21}\right)
$$$$
K_{47}=4\left(r_{23}-r_{22}-r_{13}+r_{12}\right)
$$$$
K_{49}=2\left(\left(r_{12}-r_{22}\right) x_{32}+\left(r_{13}-r_{23}\right) x_{13}\right)
$$$$
K_{56}=\left(-r_{22} x_{32}-r_{12} x_{21}\right) y_{32}+\left(-r_{12} x_{32}-r_{11} x_{21}\right) y_{21}
$$$$
K_{58}=r_{22} y_{32}^{2}+\left(r_{12} y_{21}+r_{23} y_{13}\right) y_{32}+r_{13} y_{13} y_{21}
$$$$
K_{66}=r_{22} x_{32}^{2}+2 r_{12} x_{21} x_{32}+r_{11} x_{21}^{2}
$$$$
K_{68}=\left(-r_{22} x_{32}-r_{12} x_{21}\right) y_{32}+\left(-r_{23} x_{32}-r_{13} x_{21}\right) y_{13}
$$$$
K_{77}=4\left(r_{33}-r_{23}-r_{23}+r_{22}\right)
$$$$
K_{79}=2\left(\left(r_{22}-r_{23}\right) x_{32}+\left(r_{23}-r_{33}\right) x_{13}\right)
$$$$
K_{89}=\left(-r_{22} x_{32}-r_{23} x_{13}\right) y_{32}+\left(-r_{23} x_{32}-r_{33} x_{13}\right) y_{13}
$$

The same stifhess expression applies for $K_{h a}$, if one sets $\lambda_{12}=\lambda_{23}=\lambda_{31}=\frac{1}{2}$. 
